\title{
Upregulated functionality of mitochondria-associated ER membranes in a mouse model of traumatic brain injury
}

\author{
Rishi R. Agrawall, ${ }^{1,2}$, Delfina Larrea, ${ }^{2,3}$, Lingyan Shi ${ }^{\sharp \#, ~ D o n g h u i ~ S o n g ~}{ }^{4}$, Yimeng $\mathrm{Xu}^{5}$, Taekyung D. Yun ${ }^{2,3}$, \\ Valentina Emmanuele ${ }^{2,3}$, Leslie G. Cummins ${ }^{6}$, Frank P. Macaluso ${ }^{6}$, Steven G. Kernie ${ }^{3,7}$, Wei Min ${ }^{4}$, \\ Richard J. Deckelbaum ${ }^{1,7}$, Estela Area-Gomez ${ }^{1,2,3^{*}}$
}

1. Institute of Human Nutrition, Columbia University Irving Medical Center, New York, NY 10032

2. Center for Motor Neuron Biology and Disease, Columbia University Irving Medical Center, New York, NY 10032

3. Department of Neurology, Columbia University Irving Medical Center, New York, NY 10032

4. Department of Chemistry, Columbia University, New York, NY 10027

5. Biomarkers Core Laboratory, Department of Pathology and Cell Biology, Columbia University Irving Medical Center, New York, NY 10032

6. Analytical Imaging Facility, Albert Einstein College of Medicine, Bronx, NY 10461

7. Department of Pediatrics, Columbia University Irving Medical Center, New York, NY 10032

\# Current address: Department of Bioengineering, University of California San Diego, La Jolla, CA 92093

* Correspondence to:

Estela Area-Gomez, Ph.D.

650 W. 168 ${ }^{\text {th }}$ St., Black Building 305A, New York, NY 10032

Tel: 212-305-1009; email: eag2118@cumc.columbia.edu 


\begin{abstract}
Traumatic brain injury (TBI) is a major cause of death and disability in the United States. A history of TBI can lead to neurodegenerative diseases such as Alzheimer's disease (AD) in a severity- and frequency-dependent manner. We previously reported that early stages of $A D$ are characterized by alterations in lipid metabolism due to upregulated functionality of mitochondria-associated ER membranes ("MAM" domains of the ER), a cellular hub of lipid metabolic regulation. This can be caused by increased localization of amyloid precursor protein (APP)'s C-terminal fragment of 99 a.a. (C99) at MAM, which promotes cellular cholesterol uptake and trafficking to the ER. Through this mechanism, MAM-localized C99 can stimulate MAM functionality. In this study, we recapitulate these phenotypes in the controlled cortical impact $(\mathrm{CCl})$ model of TBI in adult mice. Specifically, we observed increased phospholipid synthesis, sphingomyelinase activity and cholesterol esterification in the cortex and hippocampus 1, 3 and 7 days after injury. These responses were predominant in microglia, and coincided with increased levels of MAM-localized C99. Altogether, we propose that upregulation of MAM functionality could contribute, in part, to the epidemiological connection between TBI and AD.
\end{abstract}




\section{Introduction}

In the United States, traumatic brain injury (TBI) is the leading cause of death and disability for people under the age of 45 , and incurs an annual economic cost of $\$ 76.5$ billion. According to the CDC, 2.5 million people visited the emergency room following a TBI in 2014 , with 56,800 deaths and approximately 80,000 of these patients developing long-term disabilities (statistics sourced from Brain Trauma Foundation and $(\underline{C D C})$. A large subset of these cases is characterized by the development of neurodegenerative diseases, especially in patients who have had multiple TBls [1]. Even though these downstream consequences are usually grouped into a condition known as chronic traumatic encephalopathy (CTE) [2], a large percentage is clinically equivalent to Alzheimer's Disease (AD) [3]. Generally, physical damage is restricted to the outer, cortical layer of the brain; however, the most common neurological symptoms relate to hippocampal functions [4], which is also where $A D$ pathogenesis is thought to originate [5]. Thus, the relationship between $T B I$ and $A D$ is a growing field of research. While the epidemiological connection is established, the molecular mechanism by which a history of TBI can lead to permanent AD-like neurodegeneration is unknown.

Current understanding of TBI pathogenesis largely derives from studies in rodent models where injury parameters can be well controlled. A major focus of $T B I$ research is the recapitulation of $A D$ phenotypes in order to find shared aberrations in signaling cascades. Indeed, cardinal AD features such as amyloid- $\beta(A \beta)$ deposition and tau hyperphosphorylation, alongside neurofunctional deficits, have been reported in TBI models [6,7]. These studies suggest that TBI induces alterations in the cleavage of amyloid precursor protein (APP) similar to those found in AD, but analyses in port-mortem TBI tissues have failed to demonstrate a correlation between $A \beta /$ tau burden and disease progression [8].

An important aspect of TBI pathogenesis is its biphasic nature [9]. A primary phase of necrotic cell death at the site of injury is followed by a secondary phase of metabolic dysfunction in the surrounding area, including $\mathrm{Ca}^{2+}$ elevations, mitochondrial alterations and hypoxia [10]. The magnitude and duration of this latter phase are dependent on the severity of the injury. In most cases, these alterations will normalize; however, it is believed that, following multiple injuries, these metabolic alterations will be sustained and become chronically dysregulated. Since the likelihood of developing AD is highest among individuals who have experienced multiple TBIs, perhaps one of these chronically activated metabolic pathways underlies the connection between TBI and AD pathogenesis.

In relation to these metabolic alterations, our group and others have observed dysregulation of lipid metabolism during early stages of $A D$ [11] that resemble lipid metabolic alterations reported in neural injury models $[12,13]$. Specifically, we have observed that alterations in the activity of $\gamma$-secretase, which cleaves the amyloid precursor protein (APP) C-terminal fragment of 99 a.a. (C99) to generate $A \beta$, in AD samples result in increased ER-localized C99 [14]. Via its capacity to directly bind cholesterol, C99 can induce cholesterol trafficking to the ER and the formation of intracellular lipid rafts known as mitochondriaassociated ER membranes ("MAM" domains of the ER) [15]. The formation of MAM domains activates the recruitment of specific lipid-binding enzymes to facilitate protein-protein interactions and the modulation of 
their activities [16]. Therefore, MAM formation changes the lipid milieu of the ER, resulting in a "signaling" platform for the regulation of metabolic pathways. Increased MAM-localized C99 leads to upregulated functionality of MAM domains, which we have observed in both familial (FAD) and sporadic (SAD) AD samples [17]. This causes the dysregulation of overall cellular lipid homeostasis due to increased synthesis and turnover of specific lipid species [16]. Given the lipid metabolic alterations reported in TBI models, and the known activation of lipid synthesis and trafficking for repair of damaged membranes after brain injury [18], we hypothesized that TBI induces the activation of MAM functionality. While cellular lipid homeostasis may be restored after a single TBI episode, multiple TBIs would lead to chronic metabolic alterations that resemble $A D$, leading to $A D$-like cellular phenotypes.

In this work, using the controlled cortical impact $(\mathrm{CCl})$ model of $\mathrm{TBI}$ in adult mice, we report upregulated functionality of MAM domains during the acute phase following a single, moderate injury. This correlates with increased localization of C99 to MAM domains, and results in relevant alterations in the lipidome of brain tissues and purified microglial, astrocytic and neuronal populations. Through this preliminary study, we propose a novel pathway that could underlie the relationship between TBI and AD. 


\section{Results}

\section{The controlled cortical impact (CCl) model of TBI}

The multiple experimental models of TBI that have been developed recapitulate human TBI features to varying degrees [19]. In this study, we employed the controlled cortical impact ( $\mathrm{CCl}$ ) model in adult male WT mice. During a CCI injury, a rapidly accelerating rod penetrates the cerebral cortex through an opening in the skull [20]. We selected this model on the basis of its ability to recapitulate clinical TBI features in a reproducible manner [7,21], and the ease with which we could control injury parameters [20]. We performed an injury of moderate severity where the cortex is physically impacted but subcortical structures, including the hippocampus, are not [20].

We began this study by assaying known responses to brain injury in order to determine the regional specificity of activated molecular pathways. Our studies were conducted in the ipsilateral cortex (directly injured) and ipsilateral hippocampus (below the injury), and results were compared to naïve (uninjured) samples. We collected tissues 1, 3 and/or 7 days after injury because the APP and lipid metabolic phenotypes relevant to the hypothesis under study have been previously reported at these time-points $[13,22]$. Furthermore, we sought to generate insight into whether the parameters under study were transient or sustained during the acute phase after injury.

A well-characterized phenotype in the $\mathrm{CCl}$ model is cell death, thought to be a major contributor to behavioral alterations after TBI [4]. Since the extent and distribution of cell death differs based on injury parameters, we carried out Fluorojade-C staining, which labels degenerating neurons [23], to characterize this response in our model. We observed widespread cell death in the cortex that was nearly absent in the hippocampus (Fig. 1A). Nonetheless, gene expression of astrocyte and microglia activation markers (Gfap [24] and Aif1 [25], respectively) was elevated in both regions while elevations in Cldn11 expression (indicative of oligodendrocyte activation [26]) was limited to the cortex (Fig. 1B). These results suggest that cell death is not a requirement for a brain region to respond to physical injury, as robust glial activation is observable in the hippocampus in the absence of significant cell death.

\section{MAM activity is upregulated after brain injury}

Our results in AD suggest that APP and lipid metabolic alterations lie at the heart of disease pathogenesis [11]. We observed that, via its capacity to bind cholesterol, C99 accumulation in the ER induces the formation of MAM domains by stimulating cholesterol trafficking to the ER and the formation of lipid rafts $[14,15]$. Given the role of MAM in cellular lipid regulation, we hypothesized that brain injury could induce increases in MAM-localized C99 to stimulate lipid synthesis for membrane repair [18]. In agreement with this hypothesis, we observed a marked increase in C99 levels in MAM fractions from both cortical and hippocampal tissues (Fig. 2), without alterations in the expression of $\beta$-secretase (gene: Bace1) (Fig. S1B).

We next sought to determine whether this result correlated with increases in MAM enzymatic activities. To do this, we measured phospholipid synthesis and transfer between the ER and mitochondria, 
an established proxy measure of MAM activity [27]. Briefly, phosphatidylserine (PtdSer) is synthesized by PtdSer synthase 1 (PSS-1) at MAM domains, after which it is transferred to mitochondria for decarboxylation by PtdSer decarboxylase (PISD) to form phosphatidylethanolamine (PtdEtn) [28]. Upon pulsing crude mitochondrial fractions with radiolabeled serine, we observed significantly increased production of radiolabeled PtdSer and PtdEtn in both the cortex and hippocampus compared to naïve tissues, indicating an increase in MAM activity (Fig. 3A).

As discussed above, the formation and activation of MAM domains is induced by the transport of cholesterol from the PM to the ER [17]. Cholesterol trafficking is facilitated by the activation of sphingomyelinase (SMase) enzymes [14], which hydrolyze sphingomyelin (SM), an important structural lipid that stabilizes cholesterol in membranes [29], to form ceramide. Therefore, cholesterol mobilization and MAM formation correlate with the activation of SMase [15]. To validate the increase in MAM activity after brain injury, we measured SMase activity in hippocampal homogenates from $\mathrm{CCl}$ and naïve mice and observed a significant increase compared to naïve tissues (Fig. 3B). Remarkably, among the mammalian SMases, only the expression of Smpd5, which encodes a putative mitochondria-associated neutral SMase (MA-nSMase) [30], was significantly upregulated after TBI, in both the cortex and hippocampus (Fig. 3C).

It is known that the activation of SMase activity correlates with cholesterol esterification by acylcoA:cholesterol acyltransferase 1 (ACAT1) at MAM domains [14] to form cholesteryl esters (CEs) for storage in lipid droplets (LDs) [17]. In agreement with an increase in MAM activation after brain injury, we observed increased LDs in both the cortex and hippocampus after $\mathrm{CCl}$ compared to naïve controls (Fig. 3D). We confirmed these results by staining naïve and $\mathrm{CCl}$ tissues with Oil Red $\mathrm{O}$, which identifies neutral lipid deposits [31], observing increased staining in both brain regions after injury (Fig. 3E). Finally, we imaged the vibrational energy of lipid-specific C-H bonds through Stimulated Raman Scattering (SRS) imaging one week after injury [32]. This approach revealed a clustered signal reminiscent of granular lipid deposition that was absent in naïve tissues (Fig. S2). Together, these results speak to a stimulation of MAM activity upon brain injury, and the activation of cholesterol esterification and cellular lipid trafficking.

\section{Brain lipid composition is altered after injury}

MAM is a major hub of lipid regulation in the cell [16]. Thus, increases in MAM formation result in the upregulation of MAM-resident lipid metabolic enzymes that, in turn, cause progressive changes in lipid species constituting cellular membranes. We conducted lipidomics analysis to determine the progression of MAM-driven lipid changes in the ipsilateral cortex and hippocampus at 1, 3 and 7 days after injury (Fig. 4A). Moreover, to determine the contribution of different cell types to our data, we also determined the lipidome of isolated microglia, astrocytes and neurons (Fig. S3) from both brain areas (Fig. 4B).

In all data sets, consistent with our observed indications of increased cholesterol trafficking and deposition in LDs, we observed a trend toward reductions of free cholesterol (FC), concomitant increases in $\mathrm{CE}$, and an increased ratio of CE:FC (Fig. 4A). These results are consistent with the activation of MAM [17]. In the cortex, these alterations were sustained over the week-long period following injury, while they were nearly normalized in the hippocampus after one week. In support of this increase in lipid mobilization 
after TBI, expression of Abca1, a lipid efflux transporter [33], was significantly increased in both the cortex and hippocampus (Fig. 4C). Similarly, we observed increased expression of scavenger receptors and lipoprotein receptors. For example, $C d 36$ is a scavenger receptor for free fatty acids (FAs) [34] and was robustly increased in both the cortex and hippocampus (Fig. 4D). Expression of another scavenger receptor, Scarb1 [35], was also increased in the cortex with a trend toward an increase in the hippocampus (Fig. 4D). We also assayed the expression of $L d l r$ and $L r p 1$, lipoprotein receptors that transport cholesteroland triglyceride (TG)-rich lipoproteins [36]. Expression of both genes was increased in the cortex but not in the hippocampus (Fig. 4E). Finally, we also observed increased cortical expression of Npc2 (with a trend for an increase in the hippocampus), encoding Niemann-Pick type $\mathrm{C}$ intracellular cholesterol transporter 2, which regulates the inter-membrane transport of internalized cholesterol [37] (Fig. 4E).

Interestingly, among the purified cell type-specific populations, microglia showed a robust remodeling of their lipid composition and indications of increased MAM formation after brain injury in both the cortex and hippocampus. Specifically, microglia displayed the most consistent elevations in CEs and CE:FC ratios relative to astrocytes and neurons (Fig. 4B and Fig. 5). In support of this, microglia also displayed significant reductions in SM levels with concomitant increases in ceramide, indicative of activated SMase activity (Fig. 3B). These results suggest that, following $\mathrm{CCl}$, microglia undergo the greatest degree of lipid metabolic alterations that are consistent with MAM upregulation.

In addition, microglia from $\mathrm{CCl}$ models showed a marked decrease in levels of monoglycerides (MGs) (Fig. 4B), consistent with the known activation of monoglyceride lipase (gene Mgll) during inflammation [38]. Decreases in MGs could be attributed to increased lipid synthesis and acylation with FA chains to form diglycerides (DGs) and triglycerides (TGs) to support the assembly of membranes. Indeed, $\mathrm{CCl}$ tissues displayed significant increases in DG/TG levels, suggesting increased de novo DG/TG synthesis (Fig. 4A/B). This supports the notion that lipid synthesis and storage are robustly activated upon injury. In support of this possibility, the expression of Srebf1 (encoding sterol regulatory element-binding protein 1, SREBP1, a master regulator of fatty acid synthesis [39]), Fasn (encoding fatty acid synthase, FAS) and Acaca (encoding acetyl co-A carboxylase-1, ACC1) [40] were significantly increased in the cortex with a trend for an increase in the hippocampus (Fig. 4F). Likewise, as discussed above, expression of FA scavenger receptor Cd36 was increased in both the cortex and hippocampus (Fig. 4D), potentially due to the known role of CD36 in microglial proliferation [41].

Increases in DGs and TGs were greatest in cortical microglia and hippocampal astrocytes (Fig. 4B), potentially in support of the proliferation of these glial cells. Furthermore, levels of phosphatidylcholine (PtdCho), the most abundant phospholipid species [16], were significantly increased in astrocytes and neurons. More specifically, in purified cortical and hippocampal neurons, we observed increases in PtdCho and PtdEtn species containing long polyunsaturated fatty acyl chains at multiple time points (Fig. 6), which suggests an increase in the desaturation index of neuronal membranes after injury. 


\section{Mitochondrial bioenergetics are not impaired but substrate use is altered}

Mitochondrial dysfunction is a widely reported phenotype in experimental TBI $[42,43]$. We thus sought to determine the time-course and regional variation of respiratory impairments in our TBI model by Seahorse analysis. Surprisingly, we did not observe any significant alterations in oxygen consumption in mitochondria isolated from $\mathrm{CCl}$ brains at 1, 3 or 7 days after injury in either the cortex or hippocampus (Fig. 6A/B). This was observable when assaying both complex I (Cl)-mediated respiration (indicative of pyruvate oxidation, measured in the presence of malate as a complex II inhibitor, Fig. 6A) and complex II (CII)mediated respiration (indicative of fatty acid oxidation, $\mathrm{FAO}$, measured in the presence of rotenone as a $\mathrm{Cl}$ inhibitor, Fig. 6B) [44]. We confirmed this through histological analysis of succinate dehydrogenase (SDH, full name of CII) and cytochrome C oxidase (COX, complex IV) activities [45], both of which showed no changes in staining intensity in either region at all time-points tested (Fig. 7C/D and Fig. S5A/B). We also measured the expression of the OxPhos respiratory complexes in both brain regions at these time-points, and similarly did not observe significant alterations in expression upon injury (Fig. S5F).

As a control for these assays, we measured various parameters related to mitochondrial mass. We first assayed expression of TOM20, a marker of the mitochondrial outer membrane, by western blot (Fig. 7E). Compared to naïve, we observed a slight but significant decrease in the cortex at $7 d$ and an increase in the hippocampus at $3 \mathrm{~d}$. We also determined the ratio of mitochondrial DNA (mtDNA) to nuclear DNA (nDNA) through qPCR analysis of Cox1 (encoded by mtDNA) and Gapdh (encoded by nDNA) gene quantities. Interestingly, the ratio was unchanged in the cortex but significantly decreased in the hippocampus at $3 d$ and $7 d$ (Fig. S5C). Similarly, the expression of Ppargc1a, encoding PGC-1 $\alpha$, a regulator of mitochondrial biogenesis [46], was significantly in both brain regions $3 \mathrm{~d}$ after injury (Fig. S5D).

TBI is also reported to be associated with hypoxic stress and a consequent reduction in pyruvate oxidation by mitochondria $[47,48]$. In agreement with this, the expression of Hif1a, encoding hypoxiainducible factor 1- $\alpha(\mathrm{HIF} 1 \alpha)$, and HIF target, Pdk1, which encodes pyruvate dehydrogenase kinase 1 (PDK1), were elevated in the cortex but not the hippocampus (Fig. S6). PDK1 inhibits pyruvate dehydrogenase (PDH) activity [49] and thus reduces the use of pyruvate as mitochondrial fuel, which can cause mitochondria to begin oxidizing alternative fuel sources, such as FAs. In support of this possibility, short-chain acylcarnitines (ACs), intermediate metabolites of mitochondrial FAO [50], showed significant increases in both the cortex and hippocampus at multiple time-points (Fig. 7F). However, the expression of Cpt1a, which encodes carnitine palmitoyltransferase $1 \alpha$ (CPT-1 $\alpha)$, the enzyme that enables transport of fatty acyl-coAs into the mitochondrial matrix for oxidation [51], was significantly increased only in the cortex (Fig. S5E). When lipidomics analysis of ACs was repeated in purified cell type-specific populations, we observed that while microglia and astrocytes were certainly contributing to the bulk trends, cortical and hippocampal neurons displayed the most robust increases in these AC species (Fig. 7G).

Altogether, our results suggest that elevations in MAM-localized C99 after brain injury are associated with increased MAM activity. This correlates with subsequent changes in the regulation of cellular lipid metabolism and the composition of cellular membranes. 


\section{Discussion}

The epidemiological connection between TBI and AD is well described, but the molecular mechanism by which a history of TBI results in AD phenotypes has not been determined. Our group has previously reported that $A D$ models display upregulated functionality of MAM domains. In this work, we present data that suggests that MAM functionality is similarly upregulated in a mouse model of TBI. This correlates with lipid metabolic alterations in multiple lipid classes without marked impairments in mitochondrial respiration. We report these phenotypes in the acute phase after a single injury, where we propose that MAM upregulation is a naturally activated mechanism to promote lipid synthesis for membrane repair [52].

Alterations in APP metabolism, a cardinal feature of $A D$, have been the subject of multiple TBI studies. Deposition of $A \beta$ can be observed in humans after TBI [53] but in some studies is only observable in a minority of samples [8]. This suggests that perhaps other APP metabolites are involved in the development of $A D$ after TBI. Indeed, we have previously published in $A D$ tissues and cell models that $A B$ precursor, C99, accumulates in the ER and induces the formation and activation of MAM domains [14]. Here, we observed that similar previously reported elevations in C99 after TBI [22,54] showed an increased localization to MAM domains 3 days after injury. This correlates with increases in MAM activity, as measured by the synthesis and transfer of phospholipids between the ER and mitochondria. In AD, we observed that C99-mediated MAM upregulation is achieved by the induction of cholesterol transport from the plasma membrane (PM) to the ER, where it is esterified by ACAT1 [15]. Consistent with this, our lipidomics analysis of TBI tissues showed progressive reductions in FC and concomitant increases in CEs, suggesting increased mobilization of cholesterol from cellular membranes. Thus, our data support an active role of $\mathrm{C99}$ and cholesterol trafficking in triggering MAM activity after TBI, similar to AD.

These cholesterol alterations were observable throughout the week-long period after injury and in all cell types. Furthermore, it is known that oleoyl-CoA, or 18:1 fatty acyl CoA, is the preferred substrate for ACAT1 [55]. Thus, 18:1 CEs can be considered a surrogate marker of MAM activation. Indeed, robust increases in 18:1 CEs are readily observable after brain injury, suggesting a significant contribution of ACAT1 to the observed increases in CEs. Nevertheless, these increases could also derive from increased activity of lecithin-cholesterol acyltransferase (LCAT). The specific contributions of these enzymes to the observed phenotypes requires further investigation.

Our findings of increased MAM-mediated cholesterol trafficking after TBI are supported by previous publications in brain and nerve injury models. Elevated CEs have been reported previously $[13,56,57]$, alongside elevations in 18:1 CE specifically [13] as well as the expression of ACAT1 [12]. There are also reports of increased expression of cholesterol transporter APOE [58-60], lipid transporter LDLR [58], and lipid efflux pump ABCA1 [59,61,62]. MAM upregulation likely contributes, in part, to these phenotypes.

Increases in the transport of cholesterol and the levels of CEs were more prominent in microglia relative to astrocytes and neurons. Pro-inflammatory microglia have been shown to display increases in CEs and LDs, in part due to the phagocytosis of myelin debris [63]. The earliest reports of lipid accumulation in nerve injury models actually found that these LDs were in brain macrophages $[64,65]$. Thus, perhaps 
microglia are responsible for the majority of the cholesterol trafficking alterations after TBI. This hypothesis is supported by our data on sphingolipid alterations after injury. Previous lipidomics studies in TBI have reported reductions in SM and increases in ceramides [13,66], speaking to elevated SMase activity. Indeed, multiple activators of SMase activity (such as tumor necrosis factor alpha [67], arachidonic acid [68] and glutamate [69]) are established components of TBI pathology. We confirmed this in our model by observing elevations in SMase enzymatic activity (Fig. 3B) and Smpd5 gene expression (Fig. 3C). Interestingly, Smpd5 is predominantly expressed in microglia relative to astrocytes and neurons [70] and, in our model, only microglia displayed the SM and ceramide alterations that are consistent with SMase activation, observable throughout the week-long period after injury (Fig. 4B). Consistent with the trends we observed in cholesterol esterification, perhaps microglia are responsible for the elevations in SMase activity we observed. Indeed, SMase activation has been shown to be required for microglia to adopt pro-inflammatory phenotypes [71]. Furthermore, there is evidence that the SMase encoded by Smpd5 is associated with MAM domains. In the single report characterizing murine Smpd5, the encoded protein co-localized with both mitochondria and ER markers [30]. Our group has also previously reported that SMase activity is enriched in MAM fractions [14]. Thus, SMase activity could be considered a direct readout of MAM activation, and perhaps MAM is upregulated to a greater extent in microglia relative to astrocytes and neurons. Given the association with relevant sphingolipid alterations with microglial activation, perhaps chronic upregulation of SMase activity after multiple TBI episodes would result in sustained inflammatory phenotypes that are also characteristic of $A D$ [72]. Of note, a human homolog of Smpd5 has not been characterized; thus, the relevance of these findings to human TBI requires further investigation.

Glial activation is also associated with TG accumulation [73], which was observable here, especially at 1 and 3 days after injury. Interestingly, when we measured gene expression of lipid efflux pumps, Abca1 (enriched in astrocytes [70]) and Abcg1 (enriched in microglia [70]), only Abca1 was elevated after TBI (Figs. 4C and S4). This suggests that lipid efflux alterations are predominant in astrocytes relative to microglia. This could be representative of enhanced delivery of TGs from astrocytes to neurons as a natural mechanism to support neuronal functionality after injury. Metabolic crosstalk between astrocytes and neurons is a well-known phenomenon in neural tissues, whereby astrocytes provide neurons with numerous substrates for energy production [74]. This can include TGs, which could be harnessed for membrane repair and energy production in neurons. Further experiments are necessary to validate this hypothesis.

Additional analysis of neuronal lipidomics showed marked alterations in the acyl chain composition of phospholipid species. Specifically, we found progressive increases in PtdCho and PtdEtn species containing polyunsaturated fatty acids (PUFAs), which have been shown to increase membrane fluidity [75]. This was observable throughout the week-long period following injury in only the hippocampus for PtdCho and in both brain regions for PtdEtn. These alterations relate to reports of membrane damage in $A D$, resulting from $S A D$-associated polymorphisms in genes regulating phospholipid transport and metabolism [76]. Increases in phospholipid desaturation are induced by the activation of specific acyl-coA synthases (ACSLs), such as ACSL4 and ACSL6, which preferentially activate PUFAs for incorporation into phospholipids [77,78]. Of note, ACSLs are MAM-resident enzymes [79]. Thus, we hypothesize that the 
activities of these ACSLs may be upregulated as a result of MAM upregulation, with additional studies being necessary to confirm this mechanism. Nonetheless, such alterations can affect neuronal functionality, as the saturation index of constituent phospholipids is a major determinant of membrane permeability and fluidity [80]. This could compromise the stability of membrane-embedded ion channels and neurotransmitter receptors, impairing neurotransmission.

Finally, despite previous reports discussing mitochondrial bioenergetic impairments after TBI [43], mitochondrial respiration was not significantly impaired in our model. However, our results suggest a partial shift in substrate preference from pyruvate oxidation to FA oxidation to power OxPhos activity, in agreement with previous reports [81,82]. Perhaps this fuel shift is a counterbalancing mechanism to prevent bioenergetic failure. Indeed, reduced mitochondrial pyruvate oxidation has been demonstrated as a mechanism of preventing excitotoxicity [83], underlying a possible reason for this shift. This is another potential functional deficit that, if sustained, could become detrimental for neuronal functionality.

In summary, we propose a model in which cortical brain injury induces MAM-driven metabolic phenotypes in both the cortex and hippocampus during the acute, week-long phase following injury. The observed upregulation of cholesterol esterification and lipid trafficking correlated with elevated MAM activity levels and localization of APP-C99 to MAM domains, especially in microglial populations. This provides a potential mechanistic basis for previously observed lipid alterations after TBI. We hypothesize that, after multiple injuries, sustained dysregulation of these pathways would become detrimental for neuronal functionality, resulting in a chronic phenotype that resembles $A D$. Our data lays the groundwork for indications of an important role for MAM functionality in mediating metabolic homeostasis in the acute phase following brain injury, as well as for new ways to think about brain injury as an environmental cause of AD. 


\section{Materials and Methods}

Mouse husbandry: All animal husbandry was conducted in accordance with the Guide for the Care and Use of Laboratory Animals published by the National Institutes of Health. Specific procedures were approved by the Institutional Animal Care and Use Committee at Columbia University (protocols ACAAAO5307 and AC-AAAY6450). WT male, C57BL/6J mice (12-16 weeks in age) were ordered from Jackson labs and housed under a $12 \mathrm{~h}$ light/12h dark cycle. Following $\mathrm{CCl}$ injury (described below), the mice were placed in post-procedure housing and monitored daily for wellness. General analgesic $(5 \mathrm{mg} / \mathrm{kg}$ carprofen) was delivered via intraperitoneal (IP) injection 20 min before injury as well as 1 day (1d) and $2 d$ after injury. Mice were sacrificed 1, 3 or 7 days after injury via cervical dislocation or transcardial perfusion.

Controlled cortical impact (CCl) injury: Twenty minutes following IP injection of general analgesic (5 mg/kg carprofen), the mouse was placed in an induction chamber and exposed to $4 \%$ isoflurane for general anesthesia (supplied via an Isoflurane Vaporizer, Summit Anesthesia Solutions) supplemented with oxygen at a flow rate of 1 liter per minute (LPM). Once breathing was stabilized and there was no response to toe pinch, head fur was clipped and the mouse was secured in a stereotactic frame to initiate surgery. Anesthesia was maintained during surgery through a nose cone supplying isoflurane (eventually reduced to $2 \%$ ) and oxygen (maintained at $1 \mathrm{LPM}$ ), verified via toe pinch every 2 min. Body temperature was maintained at $37^{\circ} \mathrm{C}$ by keeping the mouse on a heating pad (Adroit Medical Systems, Loudon, TN) throughout the procedure. The scalp was disinfected by swabbing 3 times with fresh Prevantics antiseptic $3.15 \%$ chlorhexidine gluconate/70\% isopropanol (Henry Schein, Melville, NY) wipes. Local analgesic (2 $\mathrm{mg} / \mathrm{kg}$ bupivacaine) was injected subcutaneously at the injury site and 1 drop of Puralube ophthalmic ointment (Dechra Pharmaceutics, Northwich, United Kingdom) was applied to each eye. A midline incision was made to the scalp using a scalpel and a craniectomy was performed to create a $5 \mathrm{~mm} \times 5 \mathrm{~mm}$ hole in the skull between the bregma and lambda to the left of the sagittal suture. A single cortical contusion was made to the exposed dura mater using the Impact One instrument (Leica Biosystems, Buffalo Grove, IL) with the following parameters: $4.5 \mathrm{~m} / \mathrm{s}$ velocity, $1.2 \mathrm{~mm}$ depth, $0.3 \mathrm{sec}$ dwell time. Following injury, the incision was closed with sutures and Neosporin topical antibiotic ointment was applied. The mice were monitored during recovery from anesthesia.

Cytochrome $\mathbf{C}$ oxidase (COX) activity staining: Mice were euthanized 1, 3 or 7 days after $\mathrm{CCl}$ injury (alongside age-matched naïve controls) by cervical dislocation. The brain was extracted and immediately snap-frozen in isopentane at $-40^{\circ} \mathrm{C}$ for $35 \mathrm{sec}$. Following storage at $-80^{\circ} \mathrm{C}$, the brains were sectioned at $8 \mu \mathrm{m}$ thickness using a CM3050S cryostat (Leica Biosystems, Buffalo Grove, IL). The sections were stored at $-80^{\circ} \mathrm{C}$ until ready for staining, at which point they were placed at RT for 25 min before beginning the staining protocol. The staining solution consisted of $5.2 \mathrm{mM}$ 3,3-diaminobenzidine, $0.09 \mathrm{mM}$ cytochrome $\mathrm{C}$ from equine heart, and $1.4 \mu \mathrm{M}$ catalase in $5 \mathrm{mM}$ phosphate buffer, $\mathrm{pH} 7.4$, filtered through Whatman paper. The stain was applied to the tissue for $25 \mathrm{~min}$ at $37^{\circ} \mathrm{C}$ in the dark, followed by 3 gentle and quick washes with $\mathrm{ddH}_{2} \mathrm{O}$. The slides were coverslipped using warmed glycerin jelly as mounting medium. Images were collected the next day using a Nikon Eclipse 80i brightfield microscope. 
Fluoro-Jade $\mathbf{C}$ staining: Mice were euthanized 1, 3 or 7 days after $\mathrm{CCl}$ injury (alongside agematched naïve controls) via transcardial perfusion with $40 \mathrm{~mL}$ 1X phosphate-buffered saline (PBS) followed by $80 \mathrm{~mL} \mathrm{4 \%}$ paraformaldehyde (PFA, Sigma) in 1X PBS at a rate of $10 \mathrm{~mL} / \mathrm{min}$. The brain was removed and post-fixed in $4 \%$ PFA overnight $(\mathrm{ON})$ at $4^{\circ} \mathrm{C}$ with gentle agitation. The brains were embedded in $3 \%$ agarose gel in a cubic cryomold and coronally sectioned at $50 \mu \mathrm{m}$ thickness using a VT1000S vibrating blade microtome (Leica Biosystems, Buffalo Grove, IL). The sections were rinsed in PBS ON at $4^{\circ} \mathrm{C}$, mounted onto slides and stained using the Fluoro-Jade C (FJC) Ready-to-Dilute Staining Kit for Identifying Degenerating Neurons (Biosensis, Thebarton, South Australia) following manufacturer instructions. The slides were coverslipped using DPX mounting medium (Sigma) and imaged using an Olympus IX73 inverted microscope with an X-Cite Series 120 fluorescent lamp (Lumen Dynamics, Ontario, Canada).

Lipidomics analysis (bulk): At the indicated time-points after $\mathrm{CCl}$ injury, mice were euthanized (alongside age-matched naïve controls) via cervical dislocation and the brain was immediately extracted. The ipsilateral cortex and hippocampus (and corresponding regions in naïve animals) were microdissected on ice and homogenized in a Dounce homogenizer in Vance buffer (225 mM D-mannitol, 25 mM HEPES$\mathrm{KOH}, 1 \mathrm{mM}$ EGTA, pH 7.4) with cOmplete Mini EDTA-free protease inhibitor cocktail (Sigma) added at the time of use. Protein concentration was determined using the Quick Start Bradford 1x Dye Reagent (BioRad) and Quick Start BSA Standard (Bio-Rad) in a Tecan Infinite F200 PRO spectrophotometer. Equal protein amounts of each sample $(100 \mu \mathrm{g})$ were used for lipid extraction. Lipid extracts were prepared via chloroform-methanol extraction, spiked with appropriate internal standards, and analyzed using a 6490 Triple Quadrupole LC/MS system (Agilent Technologies, Santa Clara, CA) as described previously [84]. Cholesterol and CEs were separated with normal-phase HPLC using an Agilent Zorbax Rx-Sil column (inner diameter $2.1 \AA \sim 100 \mathrm{~mm}$ ) under the following conditions: mobile phase A (chloroform:methanol:1 M ammonium hydroxide, 89.9:10:0.1, v/v/v) and mobile phase $B$ (chloroform:methanol:water:ammonium hydroxide, 55:39.9:5:0.1, v/v/v/v); 95\% A for $2 \mathrm{~min}$, linear gradient to $30 \%$ A over $18 \mathrm{~min}$ and held for $3 \mathrm{~min}$, and linear gradient to $95 \%$ A over $2 \mathrm{~min}$ and held for $6 \mathrm{~min}$. Quantification of lipid species was accomplished using multiple reaction monitoring (MRM) transitions that were developed in earlier studies [84] in conjunction with referencing of appropriate internal standards. Values are represented as mole fraction with respect to total lipid (mole percentage). Lipid mass (in moles) of any specific lipid was normalized by the total mass (in moles) of all the lipids measured [84].

Lipidomics analysis (MACS-sorted populations): Following MACS isolation of cell type-specific populations, the final pellets were snap-frozen on dry ice and stored at $-80^{\circ} \mathrm{C}$ until lipid extraction. Lipidomics analysis was conducted as described above; however, since protein concentration was not determined (due to the high concentration of BSA in the isolation buffer), quantities (moles) of the extracted lipid species were normalized by quantity (moles) of total lipid extracted to determine mole percentage.

Magnetic-activated cell sorting (MACS) isolation of microglia, astrocytes and neurons: At the indicated time-points after $\mathrm{CCl}$ injury, mice were euthanized by transcardial perfusion with $50 \mathrm{~mL} 1 \mathrm{X}$ Hanks' Balanced Salt Solution ++ (HBSS with $\mathrm{Ca}^{2+}$ and $\mathrm{Mg}^{2+}$, without phenol red or $\mathrm{Na}_{2} \mathrm{CO}_{3}$ ), $\mathrm{pH} 7.4$, at a rate of $10 \mathrm{~mL} / \mathrm{min}$. The brain was extracted and the ipsilateral cortex and hippocampus (and corresponding 
regions in naïve animals) were microdissected on ice. This step was not done in the validation experiments shown in Fig. $\mathbf{S 3}$ as those were conducted in cells isolated from the whole brain (to have enough material for all of the qPCRs). For the remainder of the protocol, MACS+ Buffer (1X HBSS++, $15 \mathrm{mM} \mathrm{HEPES}$ and $0.5 \% \mathrm{BSA}$ in $\mathrm{ddH}_{2} \mathrm{O}, \mathrm{pH} 7.4$ ) was the primary buffer in all steps. The tissue was dissociated using the Neural Tissue Dissociation Kit - Postnatal Neurons (Miltenyi Biotec, Bergisch Gladbach, Germany) following manufacturer instructions with modifications: a $37^{\circ} \mathrm{C}$ oven with tube rotator was used instead of the GentleMACS dissociator, enzymes and buffer volumes were doubled (per gram tissue); trituration was conducted with a trimmed P1000 pipet tip, and a 70- $\mu$ m Falcon cell strainer (Thermo Fisher Scientific) was used instead of the MACS SmartStrainer. For the validation qPCR experiments, a $200 \mu \mathrm{L}$ aliquot of this suspension was taken as the "unsorted" sample.

For myelin removal, Myelin Removal Beads II (Miltenyi Biotec) were used. Per $0.5 \mathrm{~g}$ starting material weight, the pellet from dissociation was resuspended in $3.6 \mathrm{~mL}$ MACS+ buffer to which $400 \mu \mathrm{L}$ beads were added. The sample was incubated at $4^{\circ} \mathrm{C}$ for $15 \mathrm{~min}$ with agitation every $5 \mathrm{~min}$. The reaction was suspended by adding 5 volumes MACS+ buffer, mixing by inversion and pelleting the cells at $300 \mathrm{xg}$ for $10 \mathrm{~min}$ at $4^{\circ} \mathrm{C}$. The pellet was resuspended in $6 \mathrm{~mL}$ MACS+ buffer and evenly distributed between two LS columns (Miltenyi Biotec) in the presence of a magnetic field (generated by the QuadroMACS magnetic separator, Miltenyi Biotec). After washing the columns with $3 \mathrm{~mL}$ MACS+ buffer three times, the flow-through was centrifuged at $300 \mathrm{~g}$ for $10 \mathrm{~min}$ at $4^{\circ} \mathrm{C}$.

For microglia isolation, the resultant pellet was resuspended in $875 \mu \mathrm{L}$ MACS+ buffer in a 5-mL epitube, to which $125 \mu \mathrm{L}$ CD11b microbeads (Miltenyi Biotec) was added. The sample was placed on a rotating wheel at $4^{\circ} \mathrm{C}$ for $30 \mathrm{~min}$, after which the tube was filled with buffer and centrifuged at $300 \mathrm{~g}$ for 10 min at $4^{\circ} \mathrm{C}$. The pellet was resuspended in $4 \mathrm{~mL}$ MACS+ buffer and divided evenly between two LS columns (Miltenyi Biotec) in the presence of a magnetic field. The columns were washed 3 times each with $3 \mathrm{~mL}$ MACS+ buffer, and the total flow-through was set aside for astrocyte isolation. The microglia were collected by removing the columns from the magnetic field, applying $5 \mathrm{~mL}$ MACS+ buffer, and using the plunger to elute the captured cells. Both the eluent and flow-through were centrifuged at $300 \mathrm{~g}$ for $10 \mathrm{~min}$ at $4^{\circ} \mathrm{C}$.

For astrocyte isolation, the pellet of the flow-through was resuspended in $700 \mu \mathrm{L}$ MACS+ buffer, to which $150 \mu \mathrm{L}$ FcR Blocking Reagent (Miltenyi Biotec) was added. The sample was placed on a rotating wheel at $4^{\circ} \mathrm{C}$ for $30 \mathrm{~min}$, followed by addition of $150 \mu \mathrm{L}$ Anti-ACSA-2 microbeads. The sample was placed on a rotating wheel at $4^{\circ} \mathrm{C}$ for $30 \mathrm{~min}$, after which the tube was filled with buffer and centrifuged at $300 \mathrm{~g}$ for $10 \mathrm{~min}$ at $4^{\circ} \mathrm{C}$. The pellet was resuspended in $4 \mathrm{~mL}$ MACS+ buffer and divided evenly between two LS columns (Miltenyi Biotec) in the presence of a magnetic field. The columns were washed 3 times each with $3 \mathrm{~mL}$ MACS+ buffer, and the total flow-through was set aside as the "neurons." The astrocytes were collected by removing the columns from the magnetic field, applying $5 \mathrm{~mL}$ MACS+ buffer, and using the plunger to elute the captured cells. Both the eluent and flow-through were centrifuged at $300 \mathrm{~g}$ for $10 \mathrm{~min}$ at $4^{\circ} \mathrm{C}$. The supernatants were removed and the pellets were stored for downstream applications.

Mitochondria DNA (mtDNA) to nuclear DNA (nDNA) ratio: Mice were euthanized 1, 3 or 7 days after $\mathrm{CCl}$ injury (alongside age-matched naïve controls) by cervical dislocation. The brain was extracted 
and the ipsilateral cortex and hippocampus (and corresponding regions in naïve animals) were microdissected on ice. The tissues were homogenized in a Dounce homogenizer in Vance buffer (225 mM D-mannitol, 25 mM HEPES-KOH, 1 mM EGTA, pH 7.4) with cOmplete Mini EDTA-free protease inhibitor cocktail (Sigma) added at the time of use. Protein concentrated was quantified, and equal protein amounts $(200 \mu \mathrm{g})$ were used for each sample. Following digestion with proteinase $\mathrm{K}(1 \mathrm{mg} / \mathrm{mL})$, DNA was extracted using a standard phenol-chloroform extraction protocol with isopropanol for precipitation and ethanol for cleaning. RNA was digested using RNase A/T1 mix (Thermo Fisher Scientific EN0551) and DNA concentration was measured using a Nanodrop 1000. Quantitative PCR was conducted under standard conditions using the reaction composition in Table 1.

Oil Red $\mathrm{O}$ staining: Mice were euthanized $3 \mathrm{~d}$ after $\mathrm{CCl}$ injury (alongside age-matched naïve controls) via transcardial perfusion with $40 \mathrm{~mL}$ 1X phosphate-buffered saline (PBS) followed by $80 \mathrm{~mL} 4 \%$ paraformaldehyde (PFA, Sigma) in 1X PBS at a rate of $10 \mathrm{~mL} / \mathrm{min}$. The brain was removed and post-fixed in $4 \%$ PFA overnight $(\mathrm{ON})$ at $4^{\circ} \mathrm{C}$ with gentle agitation, cryo-protected in $30 \%$ sucrose for $2-3 \mathrm{~d}$ at $4{ }^{\circ} \mathrm{C}$ with gentle agitation (until brains sink), snap-frozen in isopentane at $-40^{\circ} \mathrm{C}$ for $35 \mathrm{sec}$, embedded in OCT medium (Thermo Fisher Scientific) and sectioned at a thickness of $16 \mu \mathrm{m}$ using a CM3050S cryostat (Leica Biosystems, Buffalo Grove, IL). Following storage at $-80^{\circ} \mathrm{C}$, the slides were allowed to equilibrate at RT for $30 \mathrm{~min}$ and placed in a humidified chamber. 10\% formalin (Thermo Fisher Scientific) was applied for 1 min. The slides were dipped in $\mathrm{ddH}_{2} \mathrm{O}$ for $30 \mathrm{sec}$ and allowed to air-dry. In the humidified chamber, Oil Red $\mathrm{O}$ $0.5 \%$ solution in propylene glycol (Poly Scientific R\&D Corp. $\mathrm{s} 1848$ ) was applied for $1 \mathrm{~h}$, followed by three 5-min dips in $\mathrm{ddH}_{2} \mathrm{O}$. The slides were air-dried and coverslipped using Fluoromount-G (Thermo Fisher Scientific). Images were immediately collected using a Nikon Eclipse 80i brightfield microscope.

Quantitative polymerase chain reaction (qPCR; bulk): Mice were euthanized 3d after CCl injury (alongside age-matched naïve controls) and the brain was immediately extracted. The ipsilateral cortex and hippocampus (and corresponding regions in naïve animals) were microdissected on ice and immediately homogenized by pipetting and vortexing in TRIzol (Thermo Fisher Scientific). Total RNA was extracted using manufacturer instructions and tested for purity by measuring $A_{260} / A_{280}$ and $A_{260} / A_{230}$ ratios via a Nanodrop 1000 (Thermo Fisher Scientific) and for integrity by agarose gel electrophoresis and detection of bands corresponding to $28 \mathrm{~S}$ and $18 \mathrm{~S}$ ribosomal RNA (rRNA). Following digestion with RQ1 RNase-free DNase (Promega), reverse transcription was performed with the High Capacity cDNA Reverse Transcription Kit (Thermo Fisher Scientific). qPCR was conducted in triplicate in a StepOne Plus real-time PCR machine (Applied Biosystems) using TaqMan Fast Advanced Master Mix (Thermo Fisher Scientific). The expression of each gene was analyzed using pre-designed TaqMan Probes (Thermo Fisher Scientific), with Gapdh (assay ID Mm99999915_g1, VIC-MGB_PL) serving as the housekeeping gene. The assay IDs of the probes used for the experimental genes tested, all conjugated to the FAM-MGB fluorophore, are listed in Table 2. Data was analyzed using the $\Delta \Delta \mathrm{Ct}$ method.

qPCR for cell type-specific markers in MACS-sorted populations: Following collection of pellets, total RNA was immediately extracted using the RNeasy Plus Micro Kit (Qiagen). Lysis was conducted by vortexing the pellet in $350 \mu \mathrm{L}$ Buffer RLT Plus containing $\beta$-mercaptoethanol (BME) for $1 \mathrm{~min}$. 
No carrier RNA was used. Purity was determined by measuring $A_{260} / A_{280}$ and $A_{260} / A_{230}$ ratios via a Nanodrop 1000 (Thermo Fisher Scientific). Because the RNeasy Plus kit includes gDNA eliminator columns, DNase digestion was not conducted as a separate step. Reverse transcription was performed using the High Capacity cDNA Reverse Transcription Kit (Thermo Fisher Scientific). qPCR was conducted in triplicate with TaqMan Fast Advanced Master Mix (Thermo Fisher Scientific) in a StepOne Plus real-time PCR machine (Thermo Fisher Scientific). The expression of each gene under study was analyzed using pre-designed TaqMan Probes (Thermo Fisher Scientific), with Rn18s/Rn45s (encoding 18S rRNA and 45S pre-rRNA; assay ID Mm03928990_g1, VIC-MGB_PL) serving as the housekeeping gene. The assay IDs of the experimental genes tested are listed in Table 3. All probes were conjugated to the FAM-MGB fluorophore. Data from each mouse was analyzed separately. Grubbs' test $(\alpha=0.05)$ was used to identify outliers, which were then removed. GOI = gene of interest.

Phospholipid transfer assay: This assay was conducted as described in $[85,86]$. Briefly, mice were euthanized 3 days after $\mathrm{CCl}$ injury (alongside age-matched naïve controls) by cervical dislocation. The brain was extracted and the ipsilateral cortex and hippocampus (and corresponding regions in naïve animals) were microdissected on ice. The tissues were homogenized with a Dounce homogenizer in Vance buffer (225 mM D-mannitol, 25 mM HEPES-KOH, 1 mM EGTA, pH 7.4) with cOmplete Mini EDTA-free protease inhibitor cocktail (Sigma) added at the time of use. Crude mitochondria fractions were isolated as described in [85,87], protein concentration was quantified and $100 \mu \mathrm{g}$ protein was brought to a volume to $50 \mu \mathrm{L}$ in phospholipid transfer assay buffer $\left(10 \mathrm{mM} \mathrm{CaCl}_{2}, 25 \mathrm{mM} \mathrm{HEPES}-\mathrm{KOH}, 0.01 \%\right.$ Triton X-100, 0.04 $\mathrm{mM}{ }^{3} \mathrm{H}$-serine). The reactions were incubated at $37^{\circ} \mathrm{C}$ for $45 \mathrm{~min}$ and stored at $-20^{\circ} \mathrm{C}$ as needed. Lipids were extracted and resolved through thin layer chromatography (TLC), followed by excision of relevant spots and scintillation counting as described in $[85,87]$. For each experiment, the average of each species in the $\mathrm{CCl}$ group was divided by the average in the naïve group in order to generate the fold change over naïve. These numbers are represented in the graph and were used for statistical analysis.

Seahorse analysis of mitochondrial respiration: Mitochondrial oxygen consumption rate (OCR) was determined as previously described [44]. In summary, mice were euthanized 1, 3 or 7 days after $\mathrm{CCl}$ injury (alongside age-matched naïve controls) by cervical dislocation. The brain was extracted and the ipsilateral cortex and hippocampus (and corresponding regions in naïve animals) were microdissected on ice. The tissues were homogenized in $\sim 10$ volumes homogenization buffer (210 mM mannitol, $70 \mathrm{mM}$ sucrose, $5 \mathrm{mM}$ HEPES and $1 \mathrm{mM}$ EGTA) and centrifuged at $900 \mathrm{xg}$ for $10 \mathrm{~min}$ at $4^{\circ} \mathrm{C}$. The remaining supernatant was centrifuged at $9000 \mathrm{xg}$ for $10 \mathrm{~min}$ at $4^{\circ} \mathrm{C}$ and the resulting pellets were resuspended in washing buffer (210 mM mannitol, $70 \mathrm{mM}$ sucrose, $5 \mathrm{mM}$ HEPES, $1 \mathrm{mM}$ EGTA, and 0.5\% FAF-BSA, pH 7.2) and centrifuged again at $8000 \mathrm{xg}$ for $10 \mathrm{~min}$ at $4^{\circ} \mathrm{C}$. The pellets, containing mitochondria, were resuspended in mitochondrial assay solution and protein concentration was measured. For C-I experiments, $8 \mu \mathrm{g}$ of protein were added to each well and $6 \mu \mathrm{g}$ for $\mathrm{C}$-II analysis. Oxygen consumption was measured at States 2, 3, 4, and uncoupling after sequential addition of $3 \mathrm{mM}$ ADP, $4 \mu \mathrm{M}$ oligomycin, $6 \mu \mathrm{M}$ FCCP and $4.5 \mu \mathrm{M}$ Antimycin A, respectively. 
Stimulated Raman Scattering imaging: Mice were euthanized 7 days after $\mathrm{CCl}$ injury (alongside age-matched naïve controls) via transcardial perfusion with $40 \mathrm{~mL} 1 \mathrm{X}$ phosphate-buffered saline (PBS) followed by $80 \mathrm{~mL} \mathrm{4 \%}$ paraformaldehyde (PFA, Sigma) in $1 X$ PBS at a rate of $10 \mathrm{~mL} / \mathrm{min}$. The brain was removed and post-fixed in $4 \%$ PFA overnight $(\mathrm{ON})$ at $4^{\circ} \mathrm{C}$ with gentle agitation. The brains were embedded in $3 \%$ agarose gel in a cubic cryomold and coronally sectioned at $50 \mu \mathrm{m}$ thickness using a VT1000S vibrating blade microtome (Leica Biosystems, Buffalo Grove, IL). The sections were rinsed in PBS ON at $4^{\circ} \mathrm{C}$, mounted onto slides and coverslipped.

We used an inverted laser-scanning microscope (FV1200, Olympus) optimized for near-IR throughput and a 25X water objective (XLPlanN, 1.05 N.A., MP, Olympus) with high near-IR transmission for SRS imaging. A picoEMERALD system (Applied Physics \& Electronics) supplied synchronized pulse pump beam (with tunable 720-990 nm wavelength, 5-6 ps pulse width, and $80-\mathrm{MHz}$ repetition rate) and Stokes (with fixed wavelength at $1064 \mathrm{~nm}, 6$ ps pulse width, and $80 \mathrm{MHz}$ repetition rate). Stokes is modulated at $8 \mathrm{MHz}$ by an electronic optic modulator. Transmission of the forward-going pump and Stokes beams after passing through the samples was collected by a high N.A. oil condenser (N.A. = 1.4). A high O.D. bandpass filter (890/220, Chroma) is used to block the Stokes beam completely and to transmit only the pump beam onto a large area Si photodiode for the detection of the stimulated Raman loss signal. The output current from the photodiode is terminated, filtered, and demodulated by a lock-in amplifier (Zurich, HF2LI) at $8 \mathrm{MHz}$ to ensure shot-noise-limited detection sensitivity. The demodulated signal is fed into analog channel of FV1200 software FluoView 4.1a (Olympus) to form image during laser scanning at a rate of 100us per pixel. For multi-channel SRS imaging, the pump wavelength was tuned so that the energy difference between pump and Stokes matched with the vibrational frequency as described below. $\lambda_{\text {pump }}=$ $1 /\left(1 / 1064+10^{-7 *} \mathrm{v}\right)$ where $\mathrm{v}$ is the vibrational frequency in $\mathrm{cm}^{-1}$. $\mathrm{CH}$ channels were acquired at $2845 \mathrm{~cm}^{-1}$ and $2940 \mathrm{~cm}^{-1}$ and unmixed according to an earlier report [32].

Sphingomyelinase (SMase) assay: This assay was conducted as described in $[85,86]$. Briefly, mice were euthanized 3 days after $\mathrm{CCl}$ injury (alongside age-matched naïve controls) by cervical dislocation. The brain was extracted and the ipsilateral cortex and hippocampus (and corresponding regions in naïve animals) were microdissected on ice. The tissues were homogenized in a Dounce homogenizer in Vance buffer (225 mM D-mannitol, 25 mM HEPES-KOH, 1 mM EGTA, pH 7.4) with cOmplete Mini EDTAfree protease inhibitor cocktail (Sigma) added at the time of use. Sphingomyelinase activity was assayed as previously described [14]. Following protein concentration quantification, $100 \mu \mathrm{g}$ protein was assayed in $100 \mathrm{mM}$ of the appropriate buffer (Tris/glycine for $\mathrm{pH} 7.0-9.0$ or sodium acetate for $\mathrm{pH} 4.0-5.0$ ), $1.55 \mathrm{mM}$ Triton X-100, 0.025\% BSA, $1 \mathrm{mM} \mathrm{MgCl}$, and $400 \mu \mathrm{M}$ bovine brain sphingomyelin (SM) spiked with 22,000 $\mathrm{dpm}$ of $\left[{ }^{3} \mathrm{H}\right]$-bovine SM (1 nCi/sample). Reactions were carried out in borosilicate glass culture tubes at $37^{\circ} \mathrm{C}$ overnight, followed by quenching with $1.2 \mathrm{~mL}$ ice-cold $10 \%$ trichloroacetic acid, incubation at $4^{\circ} \mathrm{C}$ for $30 \mathrm{~min}$, and centrifugation at $2,000 \mathrm{rpm}$ at $4^{\circ} \mathrm{C}$ for $20 \mathrm{~min} .1 \mathrm{~mL}$ supernatant was transferred to clean tubes, $1 \mathrm{~mL}$ ether was added, the mixture vortexed, and centrifuged at 2,000 rpm for $5 \mathrm{~min}$. $800 \mu \mathrm{L}$ of the bottom phase was transferred to scintillation vials with $5 \mathrm{~mL}$ of Scintiverse BD (Fisher Scientific) and measured in a Scintillation Counter (Tri-Carb 2819TR, Perkin Elmer). 
Subcellular fractionation: Mice were euthanized 3 days after $\mathrm{CCl}$ injury (alongside age-matched naïve controls) via cervical dislocation and the brain was immediately extracted. The ipsilateral cortex and hippocampus (and corresponding regions in naïve animals) were microdissected on ice and homogenized in a Dounce homogenizer in Vance buffer (225 mM D-mannitol, 25 mM HEPES-KOH, 1 mM EGTA, pH 7.4) with cOmplete Mini EDTA-free protease inhibitor cocktail (Sigma) added at the time of use. Subcellular fractionation was conducted as previously described [87].

Succinate dehydrogenase (SDH) activity staining: Mice were euthanized 1, 3 or 7 days after $\mathrm{CCl}$ injury (alongside age-matched naïve controls) by cervical dislocation. The brain was extracted and immediately snap-frozen in isopentane at $-40^{\circ} \mathrm{C}$ for $35 \mathrm{sec}$. Following storage at $-80^{\circ} \mathrm{C}$, the brains were sectioned at $8 \mu \mathrm{m}$ thickness using a CM3050S cryostat (Leica Biosystems, Buffalo Grove, IL). The sections were stored at $-80^{\circ} \mathrm{C}$ until ready for staining, at which point they were placed at RT for 25 min before beginning the staining protocol. The staining solution consisted of $5 \mathrm{mM}$ EDTA, $1 \mathrm{mM} \mathrm{KCN}, 0.2 \mathrm{mM}$ phenazine methosulfate, $50 \mathrm{mM}$ succinic acid and $1.5 \mathrm{mM}$ Nitro Blue in $5 \mathrm{mM}$ phosphate buffer, $\mathrm{pH}$ 7.6, filtered through Whatman paper. The stain was applied to the tissue for $10 \mathrm{~min}$ at $37^{\circ} \mathrm{C}$ in the dark, followed by 3 gentle and quick washes with $\mathrm{ddH}_{2} \mathrm{O}$. The slides were coverslipped using warmed glycerin jelly as mounting medium. Images were collected the next day using a Nikon Eclipse 80i brightfield microscope.

Transmission electron microscopy: Mice were euthanized 3d after $\mathrm{CCl}$ injury (alongside agematched naïve controls) via transcardial perfusion with $40 \mathrm{~mL} 1 \mathrm{X}$ phosphate-buffered saline (PBS) followed by $80 \mathrm{~mL} 4 \%$ paraformaldehyde (PFA, Sigma) in $1 \mathrm{X}$ PBS at a rate of $10 \mathrm{~mL} / \mathrm{min}$. The brain was removed, post-fixed in $4 \%$ PFA overnight $(\mathrm{ON})$ at $4^{\circ} \mathrm{C}$ with gentle agitation, and sectioned at a thickness of $100 \mu \mathrm{m}$ using a VT1000S vibrating blade microtome (Leica Biosystems, Buffalo Grove, IL). The sections were fixed by gentle agitation for $1 \mathrm{~h}$ at RT with $2 \%$ paraformaldehyde and $2.5 \%$ glutaraldehyde in $0.1 \mathrm{M}$ sodium cacodylate buffer. They were then post-fixed with $1 \%$ osmium tetroxide followed by $2 \%$ uranyl acetate, dehydrated through a graded series of ethanol and sandwiched between Aclar sheets for embedding in LX112 resin (LADD Research Industries, Burlington VT). The area of interest was cut out, glued to a blank resin block and ultrathin $(80 \mathrm{~nm})$ sections were cut on a Leica EM Ultracut UC7, stained with uranyl acetate followed by lead citrate and viewed on a JEOL 1400 Plus transmission electron microscope at 80kv.

Western blot for APP-C99: Protein concentration was determined using the Quick Start Bradford Protein Assay Kit 1 (Bio-Rad 5000201) in a Tecan Infinite F200 PRO spectrophotometer. For each fraction, $10 \mu \mathrm{g}$ protein was combined with $1 \mu \mathrm{L} 10 \%$ Triton X-100 (Sigma) and brought to a volume of $33 \mu \mathrm{L}$ in Vance buffer (225 mM D-mannitol, 25 mM HEPES-KOH, 1 mM EGTA, pH 7.4). The ratio of $10 \mu \mathrm{g}$ Triton:1 $\mu \mathrm{g}$ protein was maintained. The sample was vortexed for $15 \mathrm{sec}$, incubated on ice for $1 \mathrm{~h}$ with occasional short vortexes, and combined with $11 \mu \mathrm{L}$ loading buffer (4X NuPAGE LDS sample buffer, Thermo Fisher Scientific, with $10 \% \mathrm{BME})$. The sample was heated at $95^{\circ} \mathrm{C}$ for 5 min and loaded into a $4-12 \%$ Criterion XT Bis-Tris Protein Gel, 12+2 well, midi format (Bio-Rad 3450123) using 1X XT MES Running Buffer (Bio-Rad 1610789). Electrophoresis was conducted at $80-120 \mathrm{~V}$. The sample was transferred to an Immuno-Blot PVDF membrane (Bio-Rad 1620177) in standard tris-glycine transfer buffer with $20 \%$ methanol and $0.04 \%$ SDS at $150 \mathrm{~mA}$ for $90 \mathrm{~min}$. The antibodies used are as follows: APP-C99 (Biolegend 805701, clone M3.2); 
APP C-terminus (Sigma A8717, identifies both C99 and C83); Erlin-2 (Abcam Ab129207) and TOM20 (Santa Cruz Sc-11415).

Western blot for TOM20 and OxPhos complexes: Mice were euthanized 1, 3 or 7 days after $\mathrm{CCl}$ injury (alongside age-matched naïve controls) by cervical dislocation. The brain was extracted and the ipsilateral cortex and hippocampus (and corresponding regions in age-matched naïve animals) were microdissected on ice. The tissues were homogenized in a Dounce homogenizer in Vance buffer (225 mM D-mannitol, 25 mM HEPES-KOH, 1 mM EGTA, pH 7.4) with cOmplete Mini EDTA-free protease inhibitor cocktail (Sigma) added at the time of use. Protein concentration was quantified and $10 \mu \mathrm{g}$ was combined with $4 \mathrm{X}$ Laemmli loading buffer in a final volume of $20 \mu \mathrm{L}$, heated at $50^{\circ} \mathrm{C}$ and loaded onto a Novex WedgeWell 4-20\% tris-glycine mini SDS-PAGE gel. Electrophoresis was conducted at $80-120 \mathrm{~V}$ using standard tris-glycine running buffer. The sample was transferred to an Immuno-Blot PVDF membrane (BioRad 1620177) in standard tris-glycine transfer buffer with 20\% methanol and 0.04\% SDS at $150 \mathrm{~mA}$ for $2 \mathrm{~h}$. The antibodies used are as follows: Total OxPhos Rodent WB antibody cocktail (Abcam ab110413); TOM20 (Santa Cruz sc-11415); $\beta$-actin (Sigma A5441); and Vinculin (Sigma V4505).

Statistical analysis: Data represent mean \pm SD. All averages are the result of three or more separate animals, as indicated in the figure legends, processed independently. No statistical test was used to pre-determine sample size. Data distribution was assumed to be normal although this was not formally tested. The statistical analysis was performed using Microsoft Excel and GraphPad Prism v9.0.0 (GraphPad Software Inc., CA, USA). Statistical significance was determined by T-test or Ordinary one-way ANOVA followed by Bonferroni's multiple comparisons test, as indicated in the figure legends, at an $\alpha=0.05$ significance level. Values of $p<0.05$ were considered statistically significant. 


\section{Acknowledgments}

We thank Patricia Washington and Stephanie Siegmund for assistance with preliminary mitochondrial assays, and Sana Chintamen for advice on the brain dissociation protocols. We also thank Kristy Brown for assistance with preliminary electron microscopy experiments. We further thank all members of the Area-Gomez, Deckelbaum and Kernie laboratories for helpful discussions. We also thank Kevin Velasco, Yacine Tensaouti and Inge Hansen for laboratory assistance. Finally, we thank Eric Schon, Cristina Guardia-Laguarta, Jorge Montesinos and Kirstin Tamucci for critical feedback on the manuscript.

This work was supported by the U.S. National Institutes of Health (R01-AG056387 to E.A.-G.; R01NS088197 to R.J.D.; S10-OD016214 and P30-CA013330 to F.P.M), the National Defense Science and Engineering Graduate Fellowship (FA9550-11-C-0028 to R.R.A) and UCSD startup funds (to L.S.).

\section{Author contributions}

Conceived the project: EA-G. Designed experiments: EA-G and RRA. Generated data for most of the experiments: RRA. Provided CCl equipment and training: SGK. Critically assisted with Seahorse analysis: DL. Collected and analyzed lipidomics data: YX, TDY, RRA and EA-G. Conducted and analyzed SRS imaging studies: LS, DS, WM, RRA, RJD and EA-G. Conducted and analyzed COX/SDH staining: VE and RRA. Conducted and analyzed EM imaging: LGC, FPM, RRA, EA-G and RJD. Wrote the manuscript: RRA. Critically edited the manuscript: EA-G and RJD. Approved final version of the manuscript: all authors. 


\section{Fiqures}

\begin{tabular}{|l|c|c|c|c|}
\cline { 2 - 5 } \multicolumn{1}{c|}{} & Fluoro-Jade C & \multicolumn{3}{c|}{ CCl, days after injury: } \\
\cline { 2 - 6 } & Naive & 1 & 3 & 7 \\
\hline \multirow{3}{*}{ Cortex } & & & & 3 \\
\hline
\end{tabular}

Fluoro-Jade C DAPI
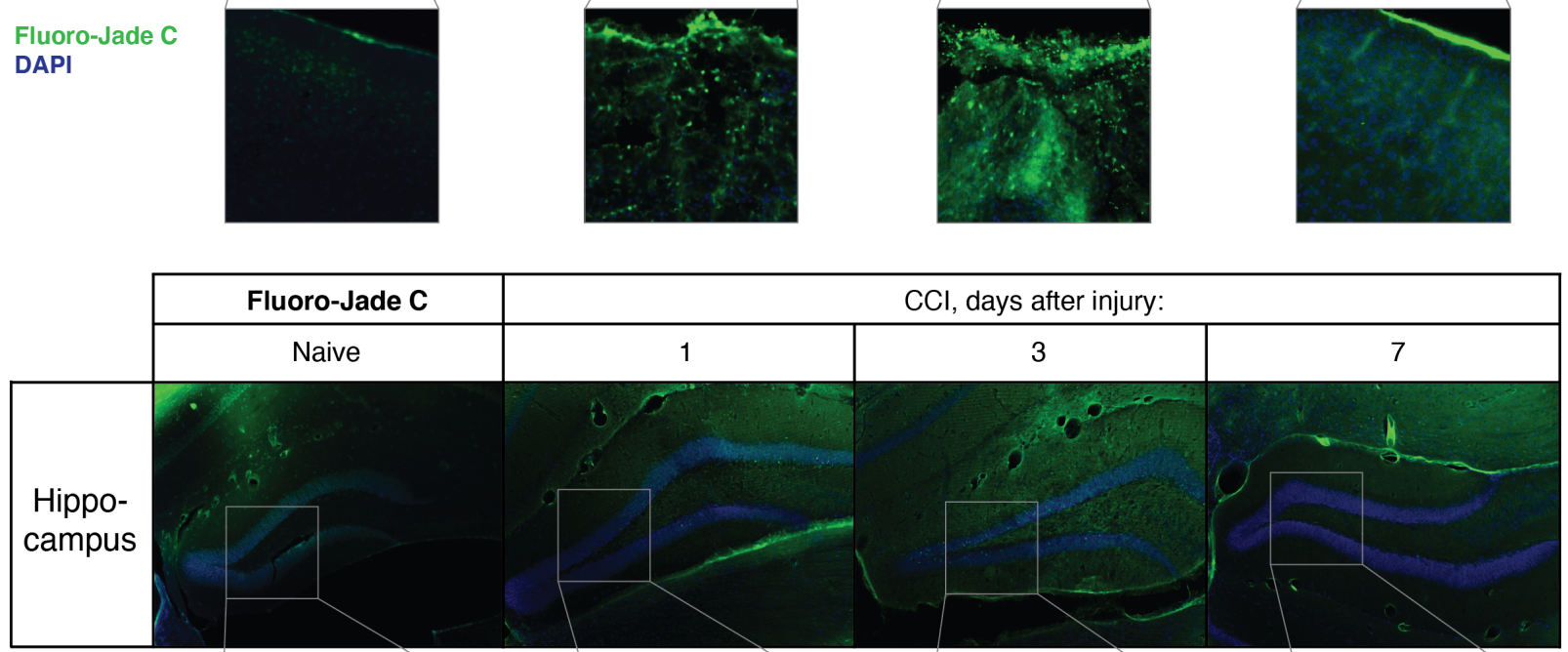

Fluoro-Jade C DAPI
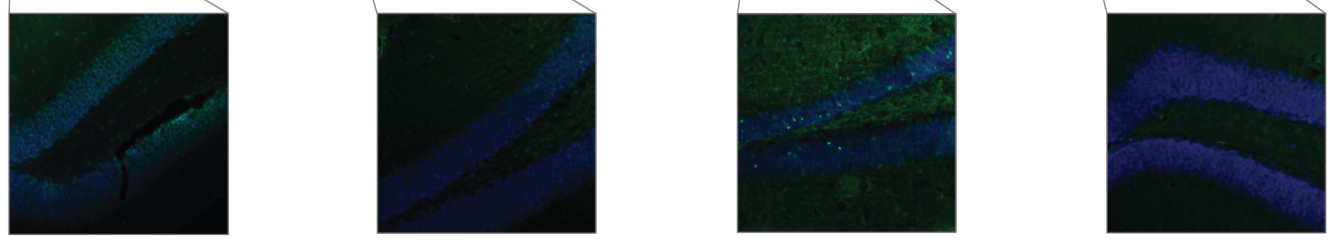

B

Gfap

C

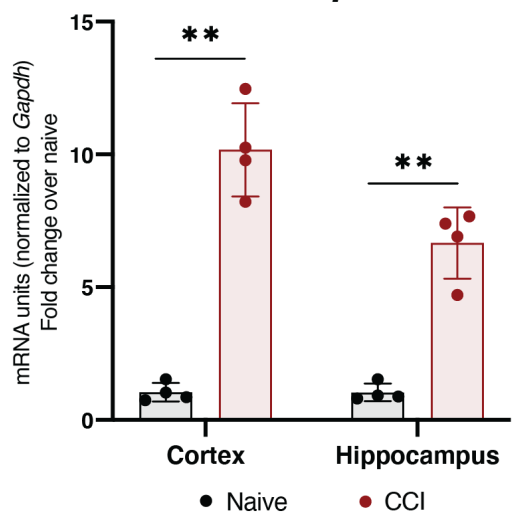

Aif1

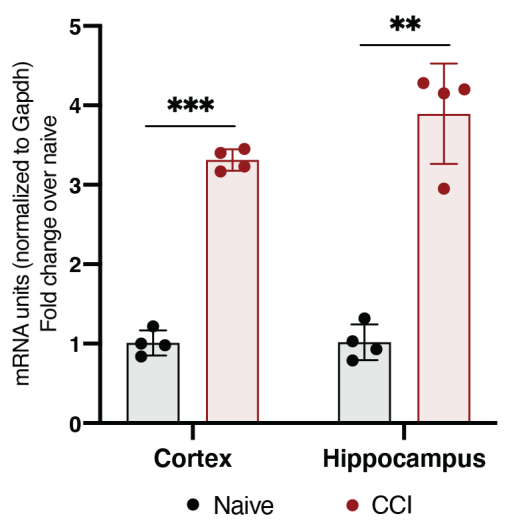

Cldn11

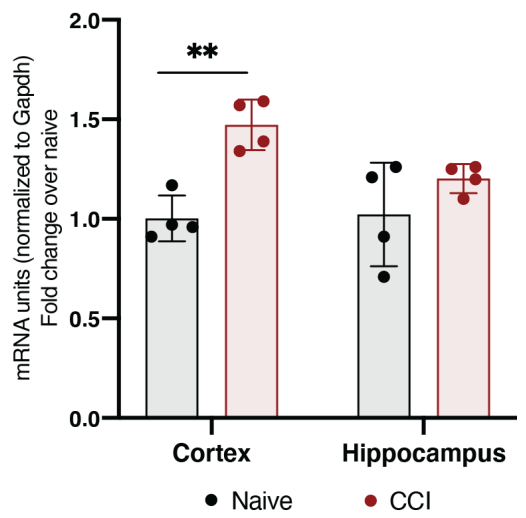




\section{Figure 1: Regional specificity of glial proliferation and cell death.}

(A) Fluoro-Jade C staining (FJC, representative of 3 independent replicates) of ipsilateral cortex and hippocampus at indicated time-points after $\mathrm{CCl}$ injury shows that neuronal degeneration occurs to a substantially greater degree in the cortex vs. the hippocampus during the post-injury period. (B-D) Activation of astrocytes and microglia in both the ipsilateral cortex and ipsilateral hippocampus 3 days after injury, and activation of oligodendrocytes onto in the cortex, normalized to Gapdh. (B) Measurement of Gfap gene expression via qPCR as an indicator of astrocytic proliferation. (C) Measurement of Aif1 gene expression (protein: IBA1) via qPCR as an indicator of microglial proliferation. (D) Measurement of Cldn11 gene expression (protein: oligodendrocyte-specific protein, OSP) via qPCR as an indicator of oligodendrocyte proliferation. Each data point represents a separate mouse (biological replicate) and is representative of 3 technical replicates (qPCR wells). Error bars represent standard deviation among biological replicates. For all assays, comparisons are made to naïve (uninjured) tissues harvested and assayed alongside $\mathrm{CCl}$ tissues. ${ }^{* *}, \mathrm{p}<0.01 ;{ }^{* * *}, \mathrm{p}<0.001$; T-test. 

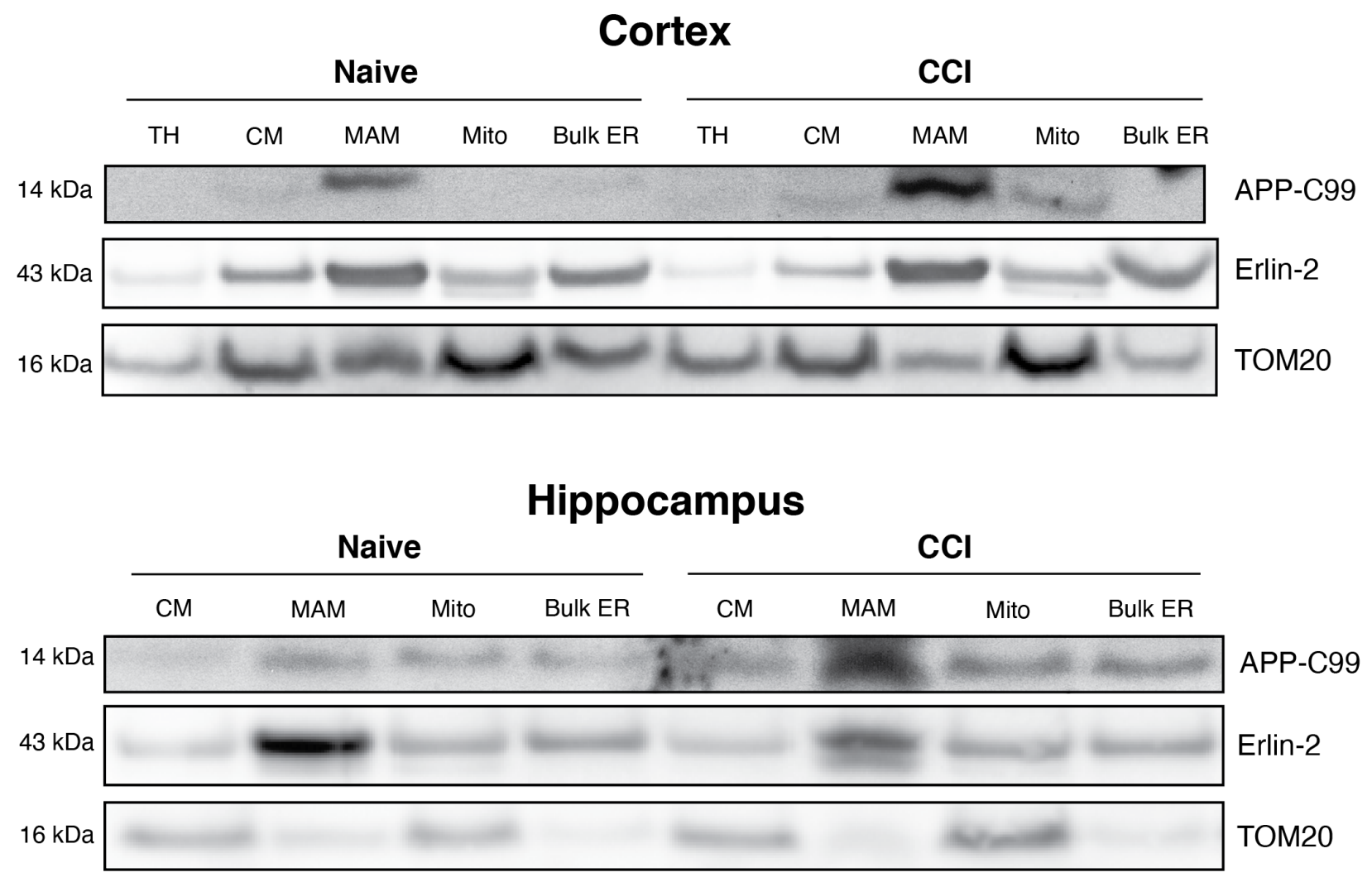

Figure 2: Localization of APP-C99 to MAM domains is increased after CCl.

Subcellular fractionation of ipsilateral cortical and hippocampal homogenates 3 days after injury shows that APP-C99 (14 kDa) levels are elevated upon injury in the MAM fraction. This is consistent with enhanced signal using an antibody for the C-terminus for APP, which would include APP-C83 (13 kDa). Erlin-2 and TOM20 serve as MAM and mitochondrial markers, respectively. This western blot is representative of 3 independent replicates. Naïve (uninjured) tissues were harvested and assayed alongside CCI tissues. CM, crude membranes fraction. 

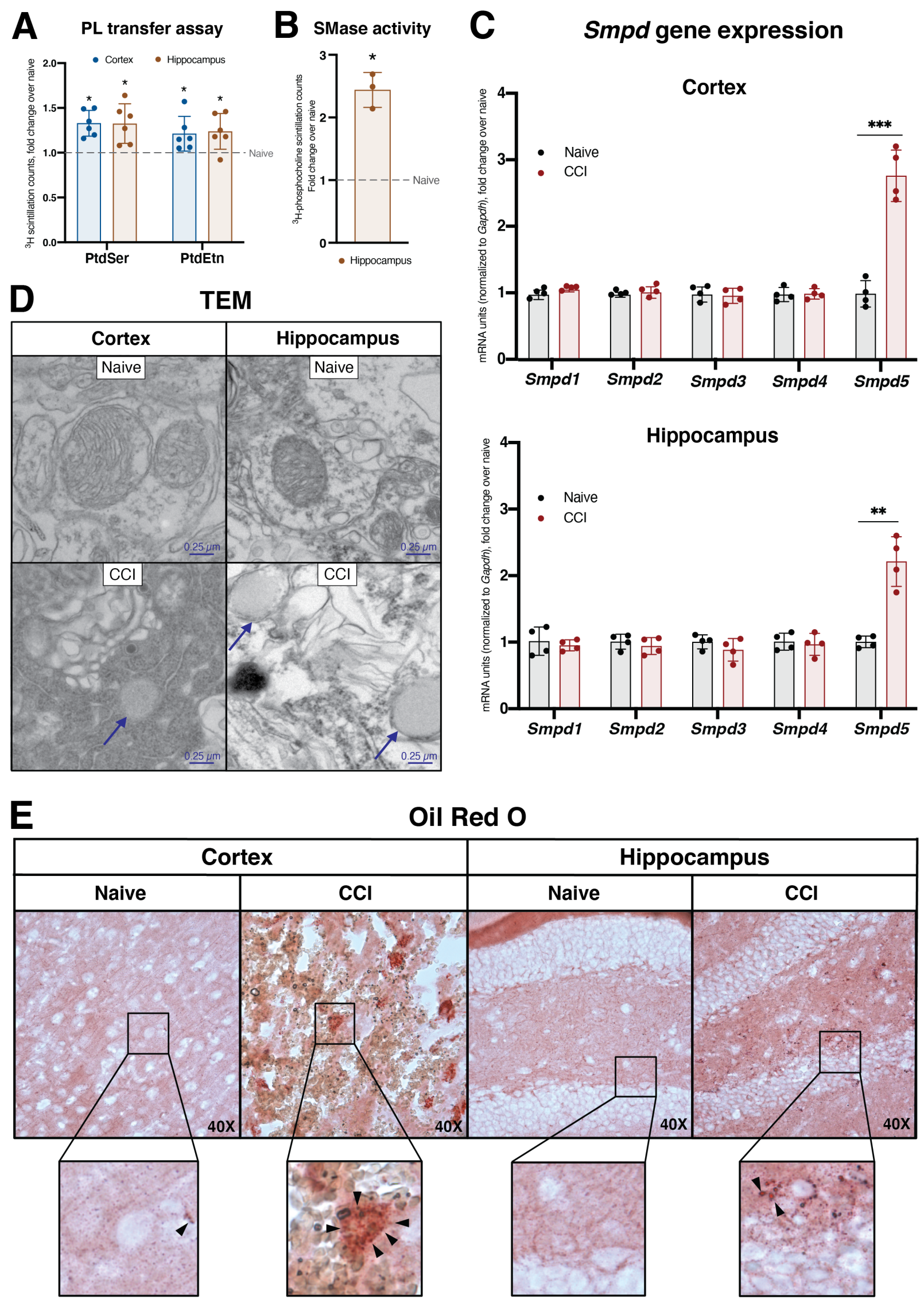
Figure 3: MAM functionality is upregulated after $\mathrm{CCl}$ in both the cortex and hippocampus.

All $\mathrm{CCl}$ samples shown in this sample are from the ipsilateral hemisphere. Naïve samples were processed alongside the $\mathrm{CCl}$ samples. (A) Analysis of phospholipid synthesis 3 days after injury shows increased synthesis of both phosphatidylserine (PtdSer) and phosphatidylethanolamine (PtdEtn), indicating increased communication between the ER and mitochondria. (B) Sphingomyelinase (SMase) activity assay showing increased SMase activity in the hippocampus 3 days after injury. (C) Gene expression analysis via qPCR of the Smpd genes, which encode the five mammalian SMase isoforms, 3 days after injury, normalized to Gapdh. Expression of Smpd5, but not the other genes, is significantly increased. Each data point represents a separate mouse (biological replicate) and the average of 3 technical replicates (qPCR wells). Error bars represent standard deviation among biological replicates. *, $p<0.05$; ${ }^{* *}, p<0.01$; T-test. (D) Representative electron microscopy image 3 days after injury with visible lipid droplets. (E) Representative Oil Red O staining of the ipsilateral cortex and hippocampus 3 days after injury shows lipid deposition in both brain regions. 

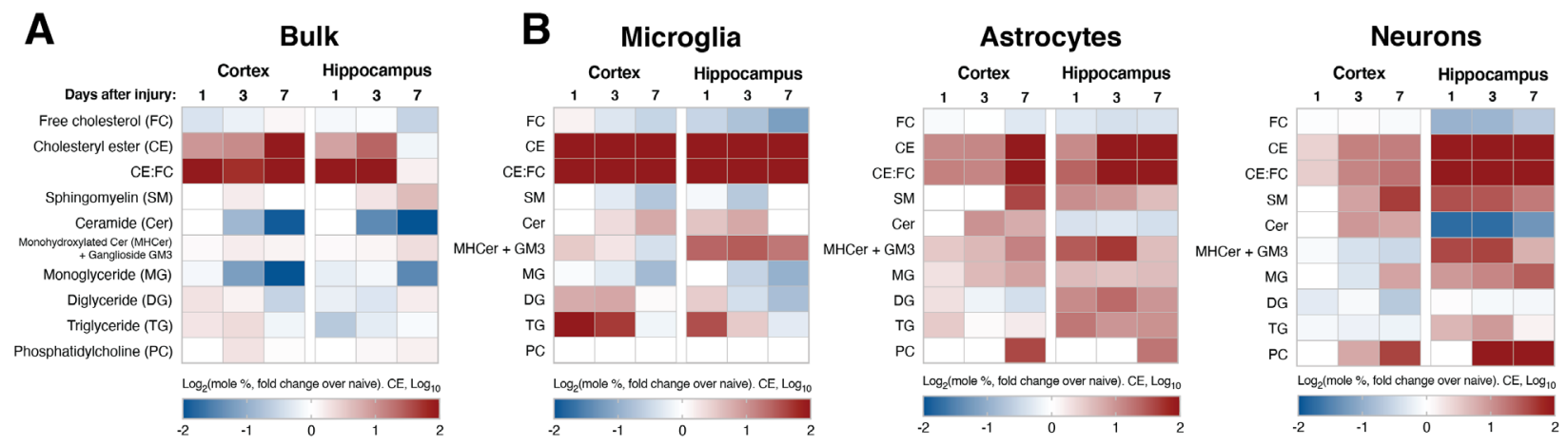

\section{Lipid efflux}

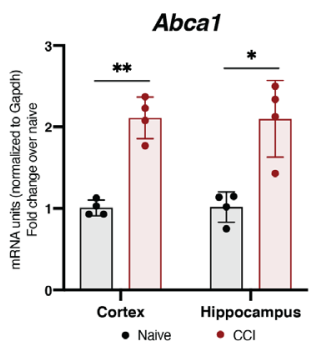

D

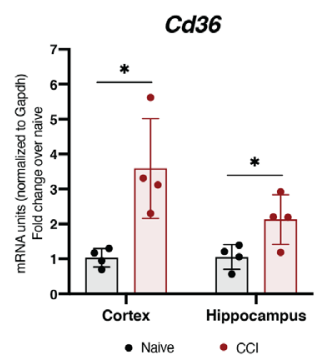

Lipid uptake

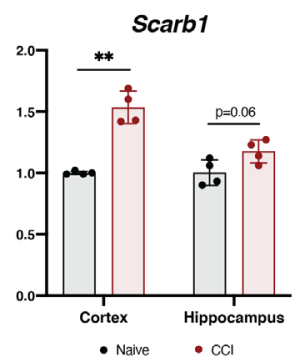

E

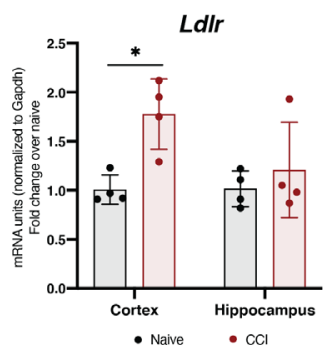

$\mathbf{F}$

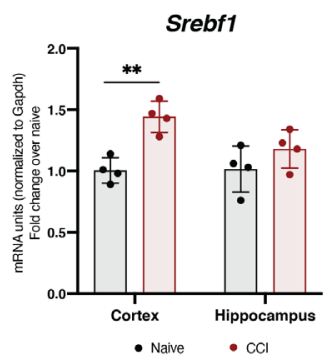

Lipoprotein uptake and hydrolysis
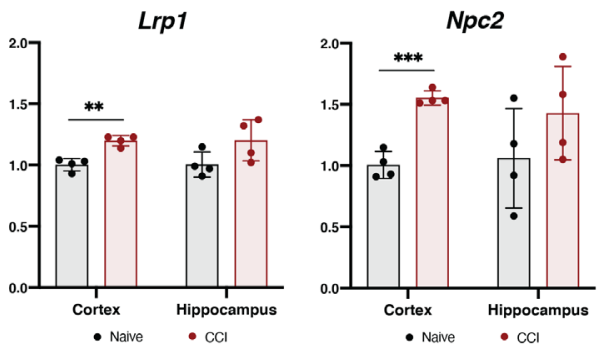

Lipid synthesis
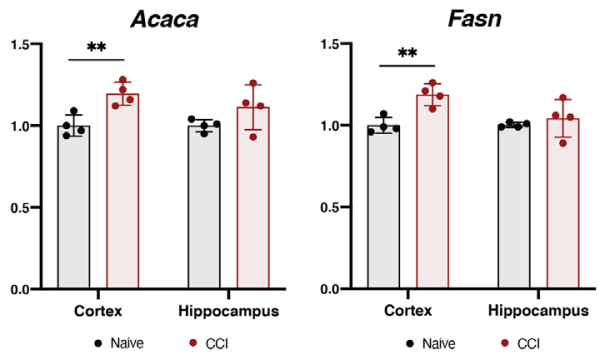

Figure 4: Elevations in cellular lipid trafficking and synthesis are observable in both bulk homogenates and purified cell type-specific populations after $\mathrm{CCl}$.

(A, B) Heatmap of fold changes of indicated lipid species at 1, 3 and 7 days after $\mathrm{CCl}$ over naïve, in (A) bulk cortical and hippocampal homogenates and (B) sorted populations of microglia, astrocytes and neurons. (C-F) Expression of genes important in cellular lipid metabolism 3 days after injury, normalized to Gapdh. Each data point represents a separate mouse (biological replicate) and the average of 3 technical replicates (qPCR wells). Error bars represent standard deviation among biological replicates. * $p<0.05 ;{ }^{* *}$, $\mathrm{p}<0.01$; T-test. (C) Increased gene expression of lipid efflux pump, Abca1, is observable in both the cortex and hippocampus. (D) Gene expression of scavenger lipid receptors, Cd36 and Scarb1. Cd36 is increased in both the cortex and hippocampus, while Scarb1 is increased in the cortex and displays a trend for an increase in the hippocampus. (E) Gene expression of mediators of lipoprotein uptake (Ldlr and Lrp1) and hydrolysis (Npc2) mediators. Increases are observable in the cortex but not the hippocampus. (F) Gene expression of Srebf1, Acaca and Fasn, regulators of de novo lipogenesis, is significantly increased in the cortex but not the hippocampus. 
A

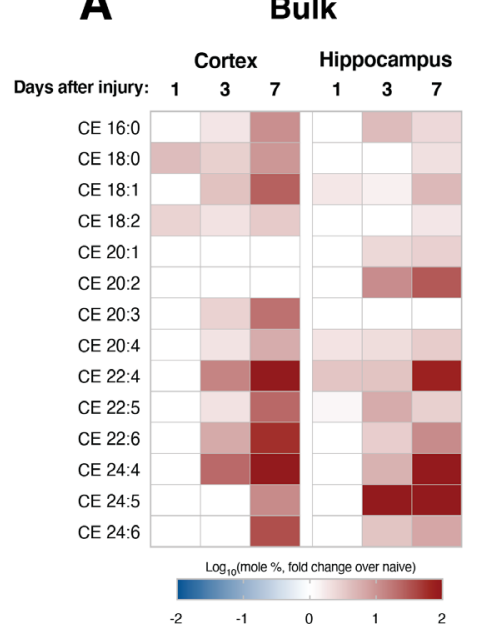

\section{B Microglia}

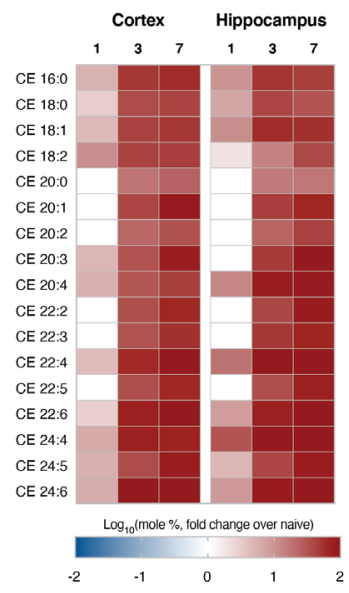

Astrocytes

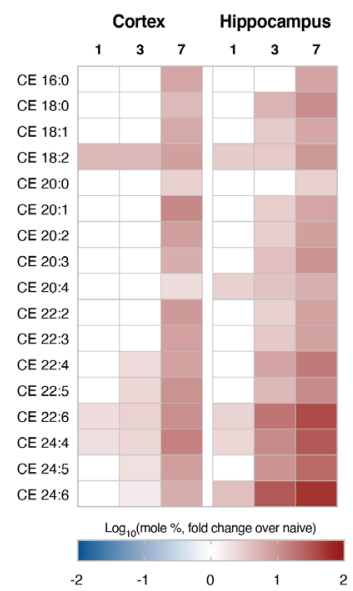

Neurons

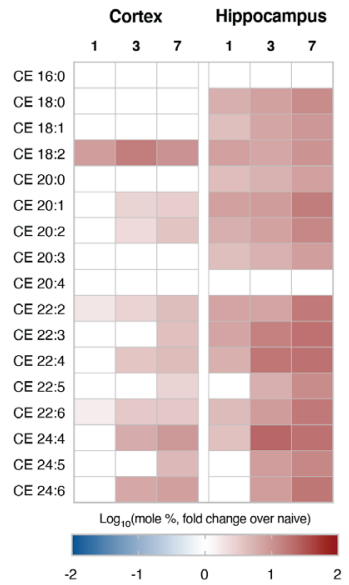

Figure 5: Lipidomics analysis of individual cholesteryl ester (CE) species indicates increased CE concentrations throughout the post-injury time course, especially in microglia.

(A) Lipidomics levels of individual CE species in bulk ipsilateral cortical and hippocampal homogenates at the indicated time-points after injury. (B) Lipidomics levels of individual CE species in sorted populations of cortical and hippocampal microglia, astrocytes and neurons at the indicated time-points after injury. 


\section{Neurons:}

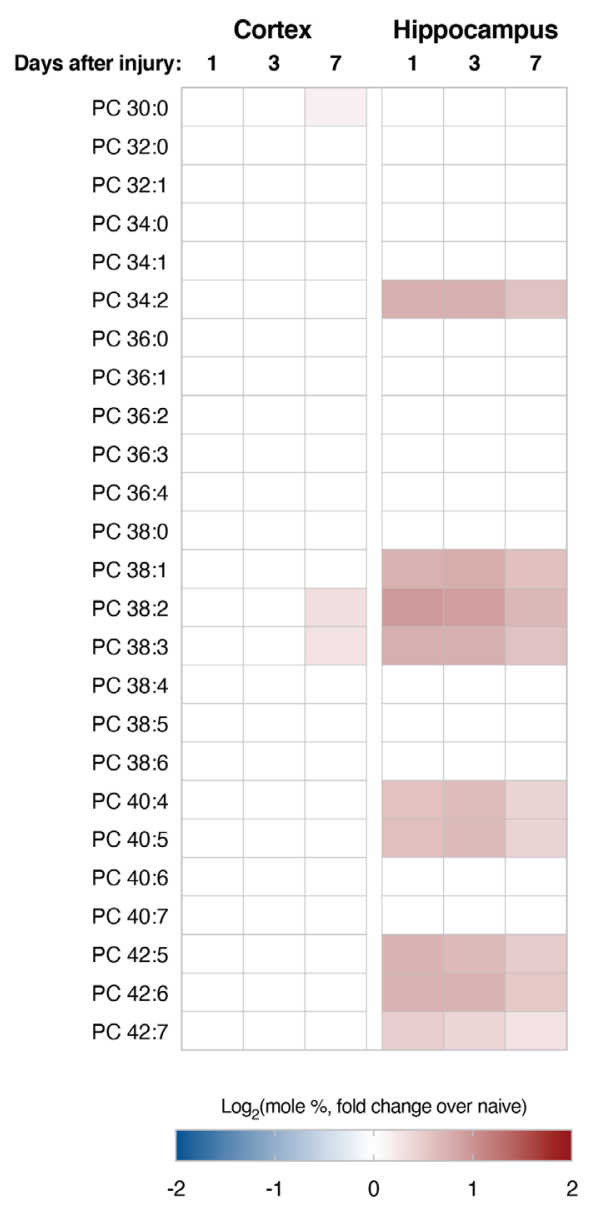

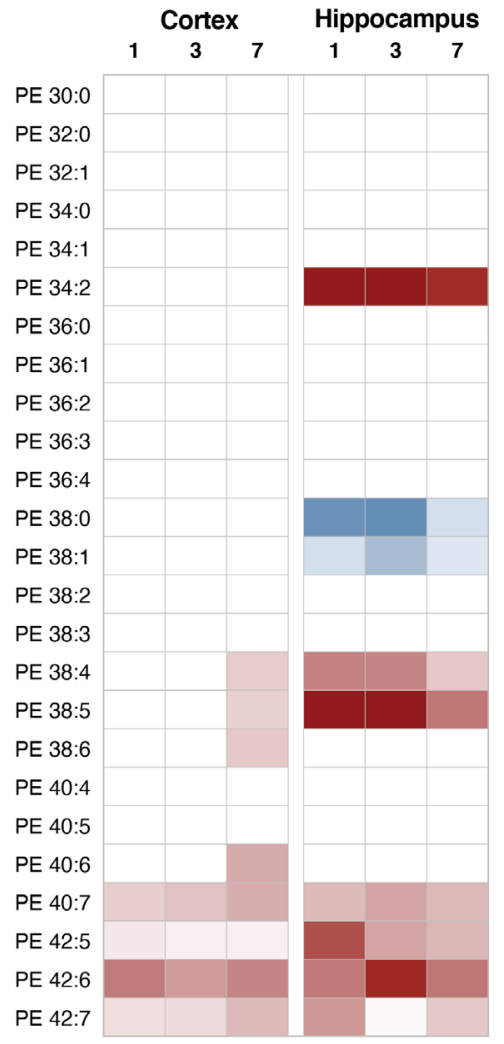

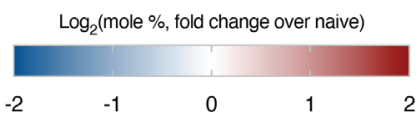

Figure 6: Increased levels of polyunsaturated PtdCho and PtdEtn species in cortical and hippocampal neurons.

Lipidomics analysis in neurons purified at indicated time points after $\mathrm{CCl}$ from the ipsilateral cortex and hippocampus. Significant increases are observable specifically in species containing polyunsaturated acyl chains. 
A
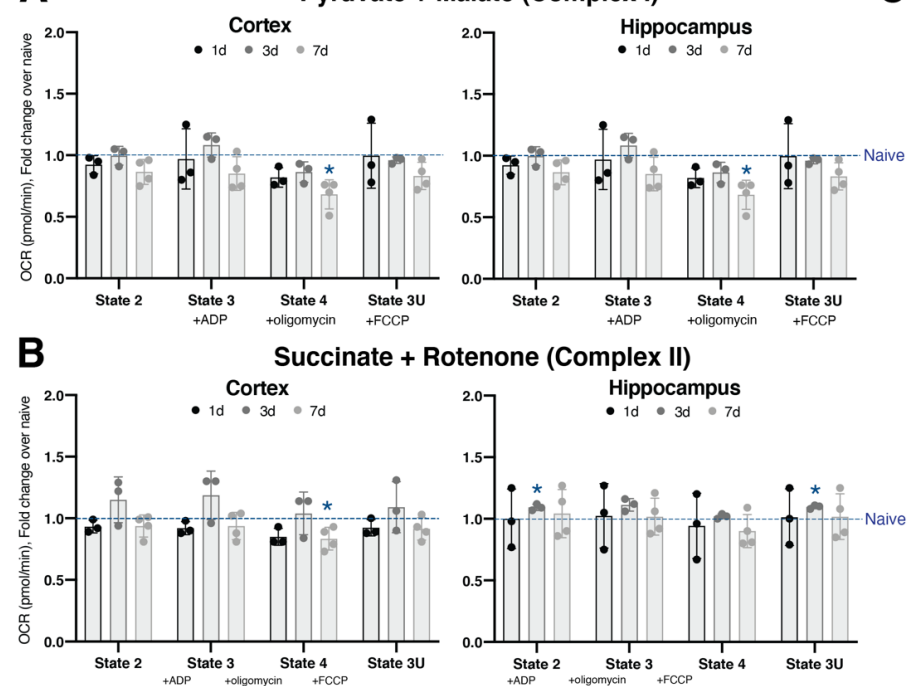

E

Cortex
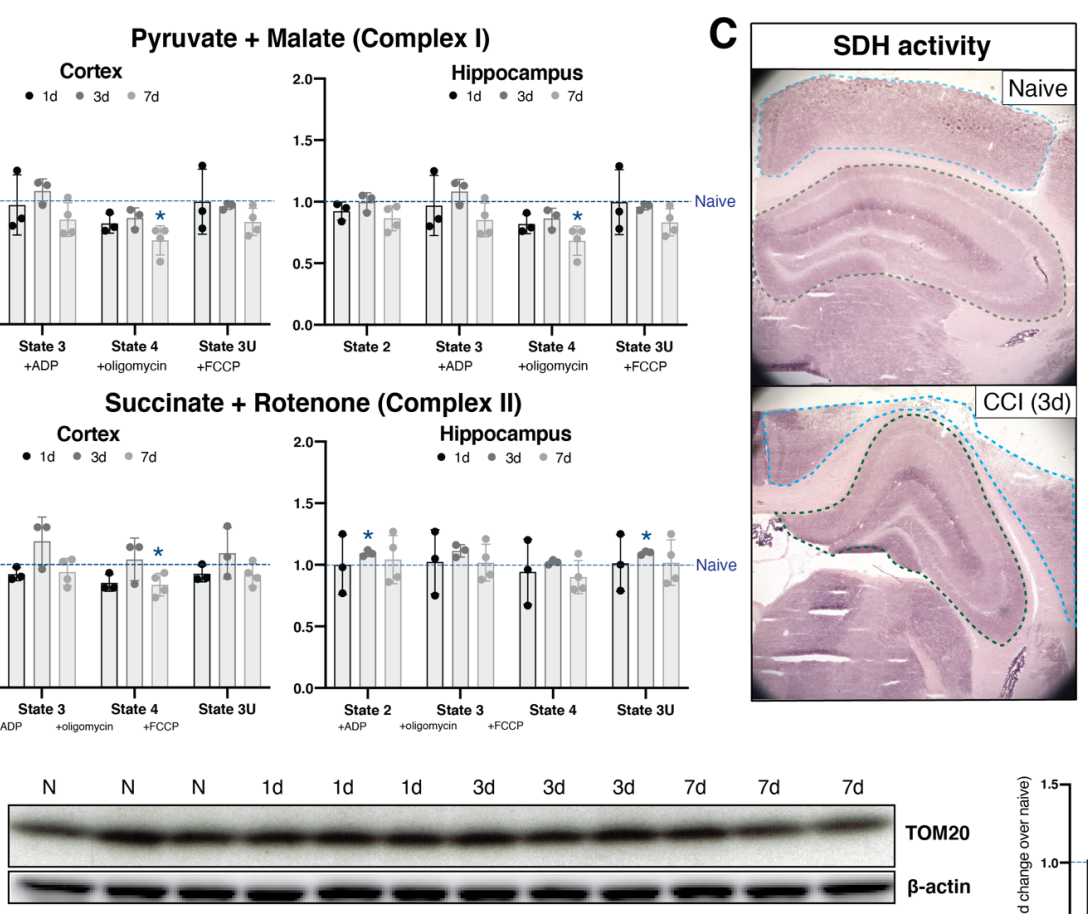

D

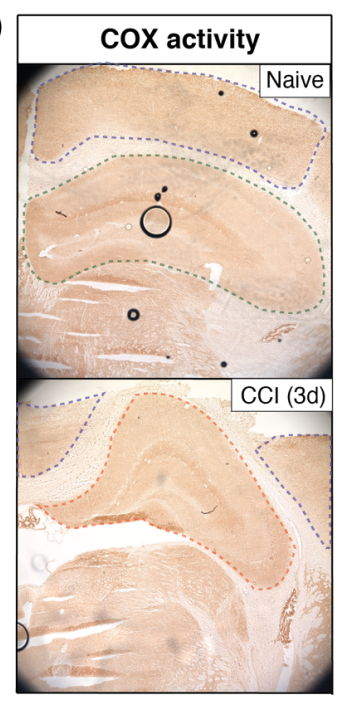

Hippo-

$\mathrm{N} \quad \mathrm{N}$
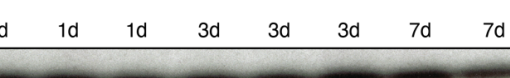

$7 \mathrm{~d}$ campus

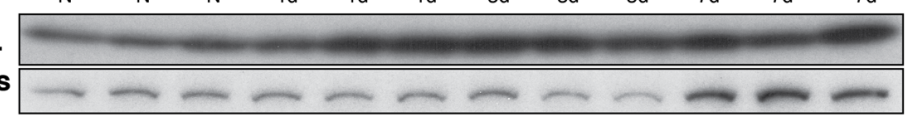
том20 Vinculin
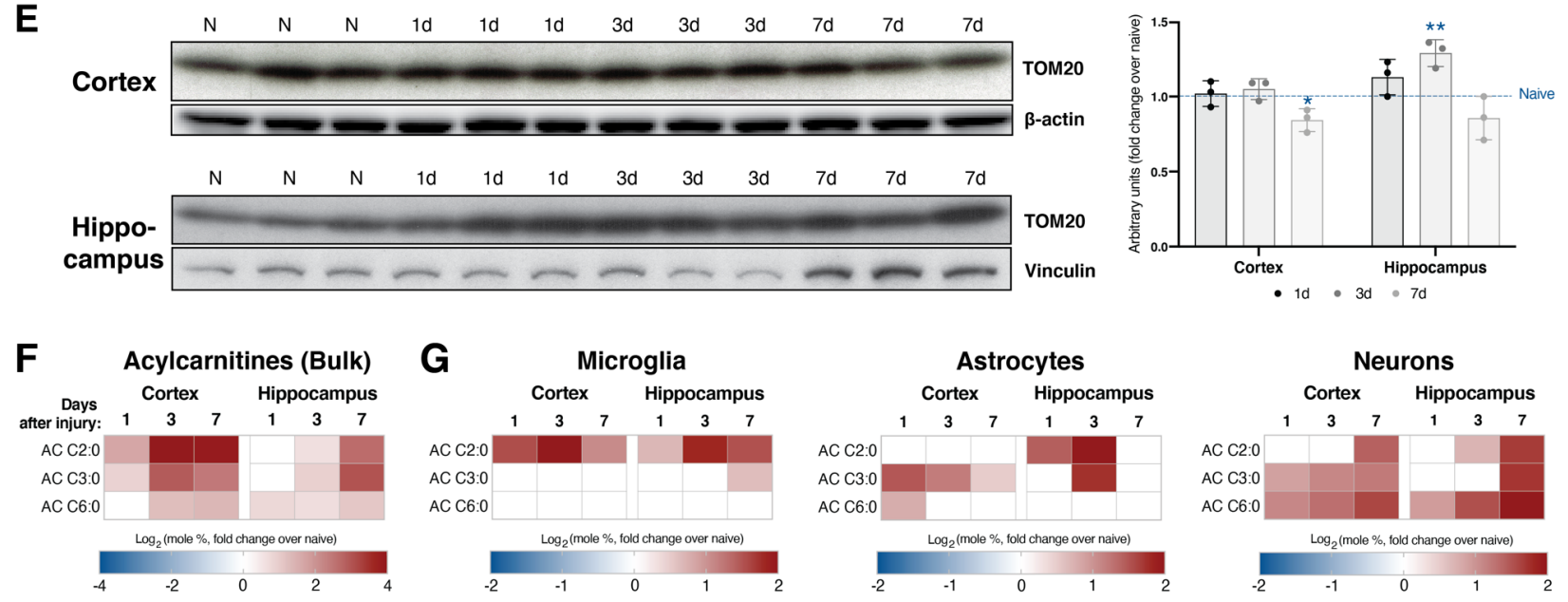


\section{Figure 7: Cortical and hippocampal mitochondria display a moderate switch in substrate preference without impairments in bioenergetics.}

$(A, B)$ Mitochondrial oxygen consumption rate $(O C R)$ measured in the Seahorse XF Analyzer in crude mitochondrial fractions collected at the indicated time-points from the indicated ipsilateral brain regions. OCR values are shown by respiratory state: State 2, baseline; State 3, after ADP addition to stimulate electron flow; State 4, after oligomycin (ATP synthase inhibitor) addition to determine the portion of oxygen consumption occurring for the purpose of ATP production; State 3-uncoupled (3U), after trifluoromethoxy carbonylcyanide phenylhydrazone (FCCP) addition in order to uncouple electron flow and oxygen consumption from ATP production. (A) Respiration in the presence of pyruvate and malate (complex-II inhibitor) in order to assay the contribution of complex-I (i.e., glucose oxidation) to cellular respiration. (B) Respiration in the presence of succinate (complex-II substrate) and rotenone (complex-I inhibitor) in order to assay the contribution of complex-II (i.e., fatty acid oxidation) to cellular respiration). Overall, mitochondrial respiration levels were not strongly affected by $\mathrm{CCl}$ injury. (C) Representative succinate dehydrogenase $(\mathrm{SDH}$, complex-II) activity staining images in the ipsilateral cortex (outlined in blue) and ipsilateral hippocampus (outlined in yellow) 3 days after CCI. (D) Representative cytochrome C oxidase (COX) activity staining images in the ipsilateral cortex (outlined in blue) and ipsilateral hippocampus (outlined in yellow) 3 days after CCI. (E) Expression of TOM20 (a marker for mitochondrial mass) in cortical and hippocampal homogenates at the indicated time-points after injury. Each lane is a separate mouse (biological replicate). $\beta$-actin is the loading control for the cortex and vinculin is the loading control for the hippocampus. Densitometry is shown as well. (F) Bulk lipidomics analysis of total acylcarnitines (ACs), which become elevated with increased fatty acid $\beta$-oxidation, shows increases in the both the ipsilateral cortex and hippocampus at multiple time points after injury. (G) Lipidomics analysis of total ACs in astrocytes and microglia from both the cortex and hippocampus. ACs are highest in both cell types at the mid-point of the injury time course, with the most stable increased in cortical astrocytes. ${ }^{*}, \mathrm{p}<0.05 ;{ }^{* *}$, $\mathrm{p}<0.01$; T-test. 

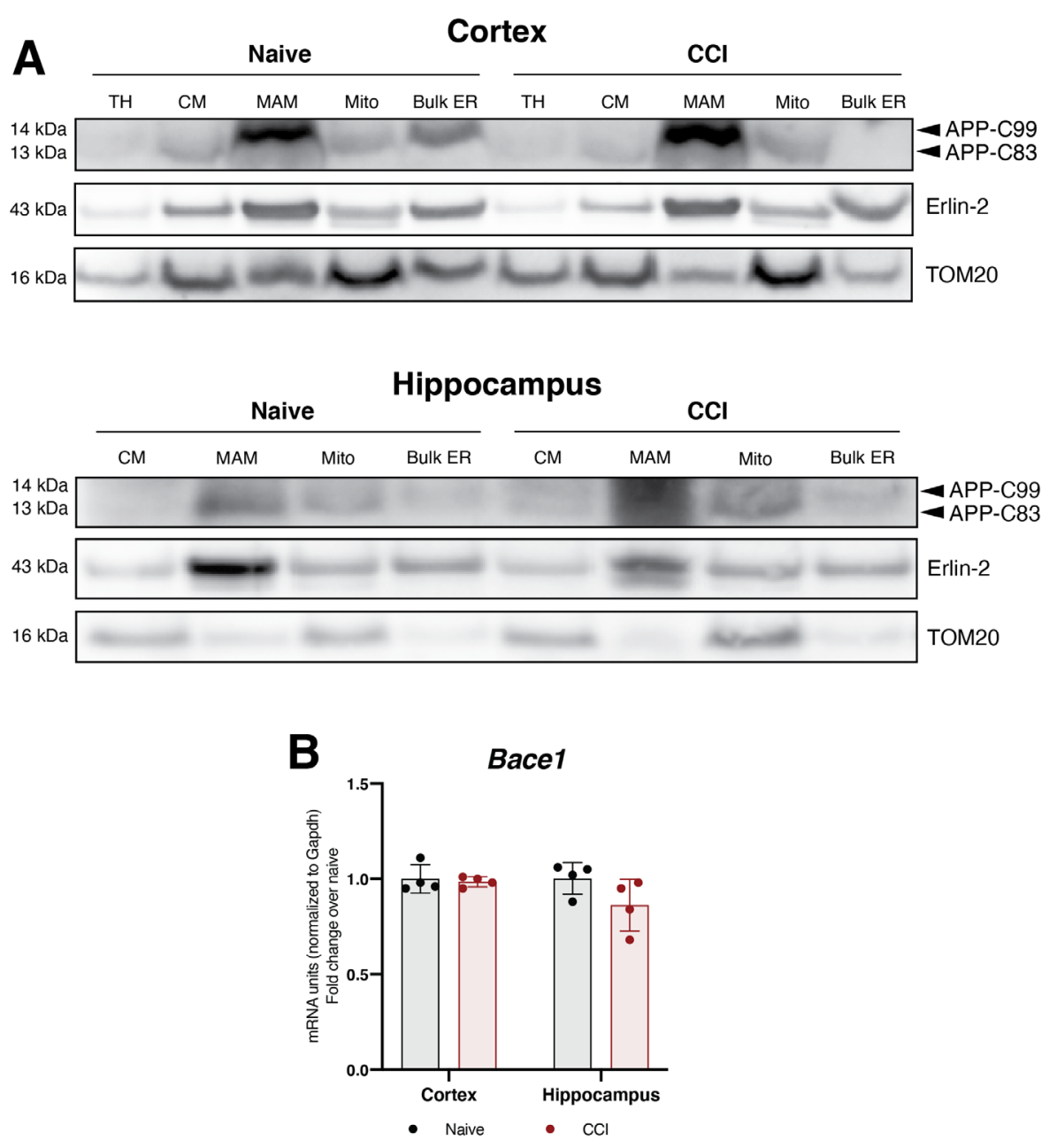

Supplemental Figure 1: Additional parameters of APP processing after $\mathbf{C C l}$.

(A) Subcellular fractionation of ipsilateral cortical and hippocampal homogenates 3 days after injury shows enhanced signal using an antibody for the C-terminus for APP, which includes both APP-C99 (14 kDa) and APP-C83 (13 kDa) upon injury in the MAM fraction. Erlin-2 and TOM20 serve as MAM and mitochondrial markers, respectively. This western blot is representative of 3 independent replicates. TH, total homogenate. CM, crude membranes fraction. (B) Gene expression analysis via qPCR of Bace1, which encodes $\beta$-secretase (the enzyme responsible for C99 production from full-length APP), 3 days after injury, normalized to Gapdh. Each data point represents a separate mouse (biological replicate) and the average of 3 technical replicates. Bace1 expression is not significantly changed in either brain region (T-test, $\alpha=0.05$ ). Naïve (uninjured) tissues were harvested and assayed alongside $\mathrm{CCl}$ tissues. Error bars represent standard deviation among biological replicates. 


\section{Stimulated Raman Scattering} (7d after injury)

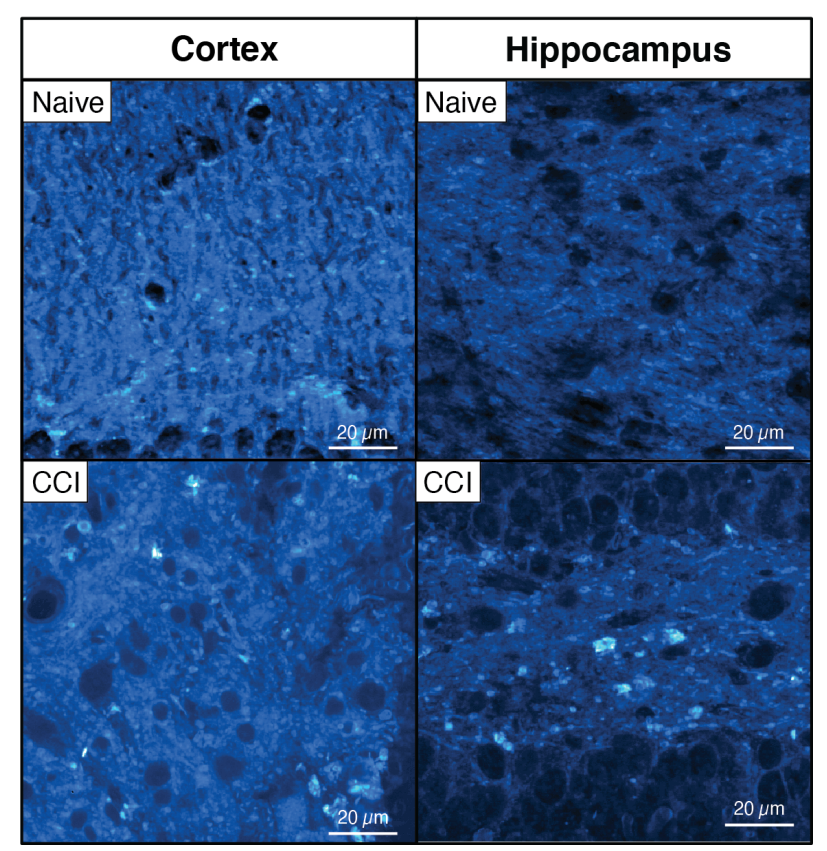

Supplemental Figure 2: Stimulated Raman Scattering (SRS) imaging of lipid-specific C-H bonds 7 days after $\mathbf{C C l}$.

Stimulated Raman scattering (SRS) microscopy image of C-H bonds in lipid channel showing increased lipid clusters 7 days after injury. 


\section{A}
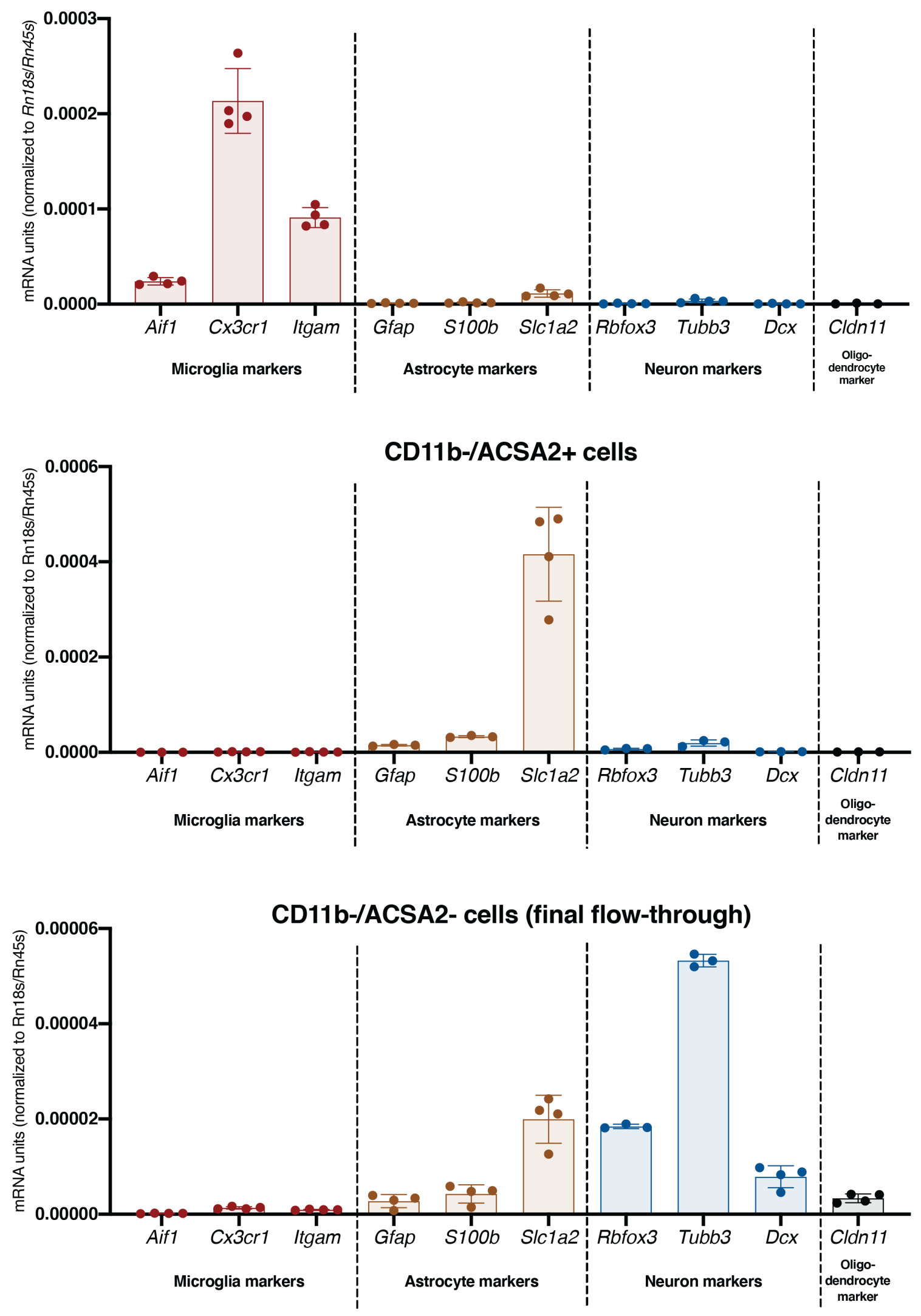
B

\section{Microglia markers:}
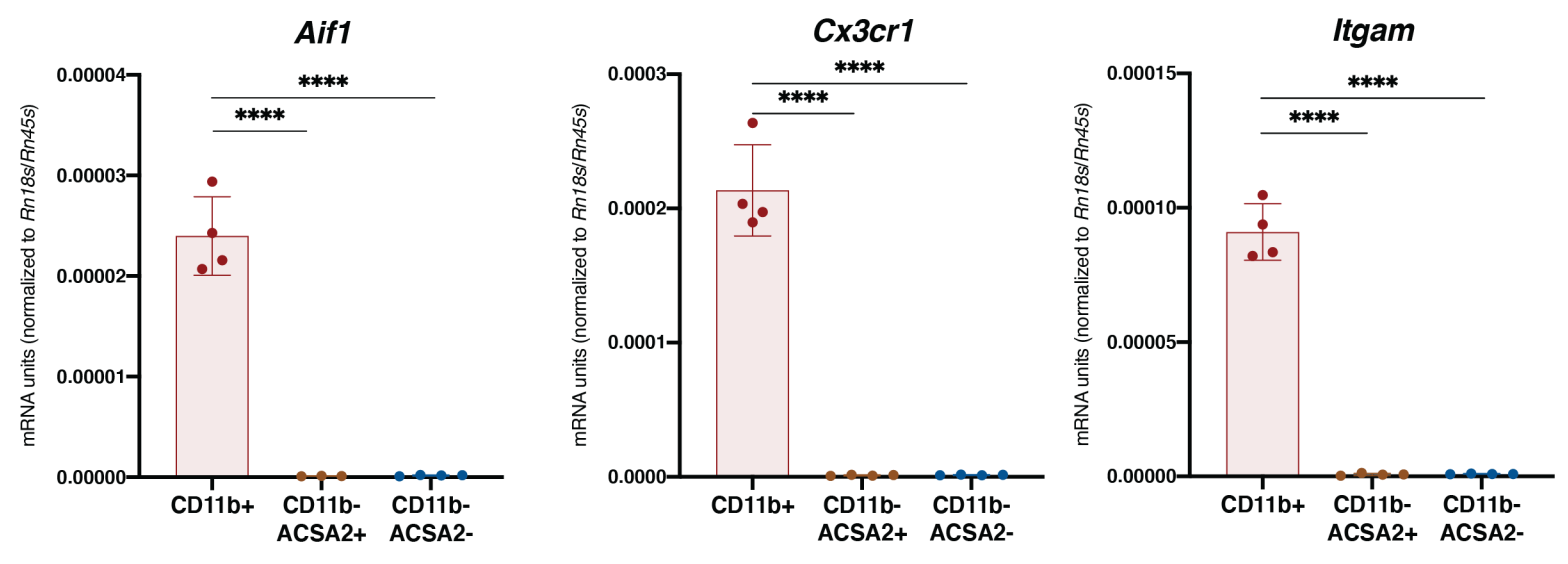

\section{Astrocyte markers:}
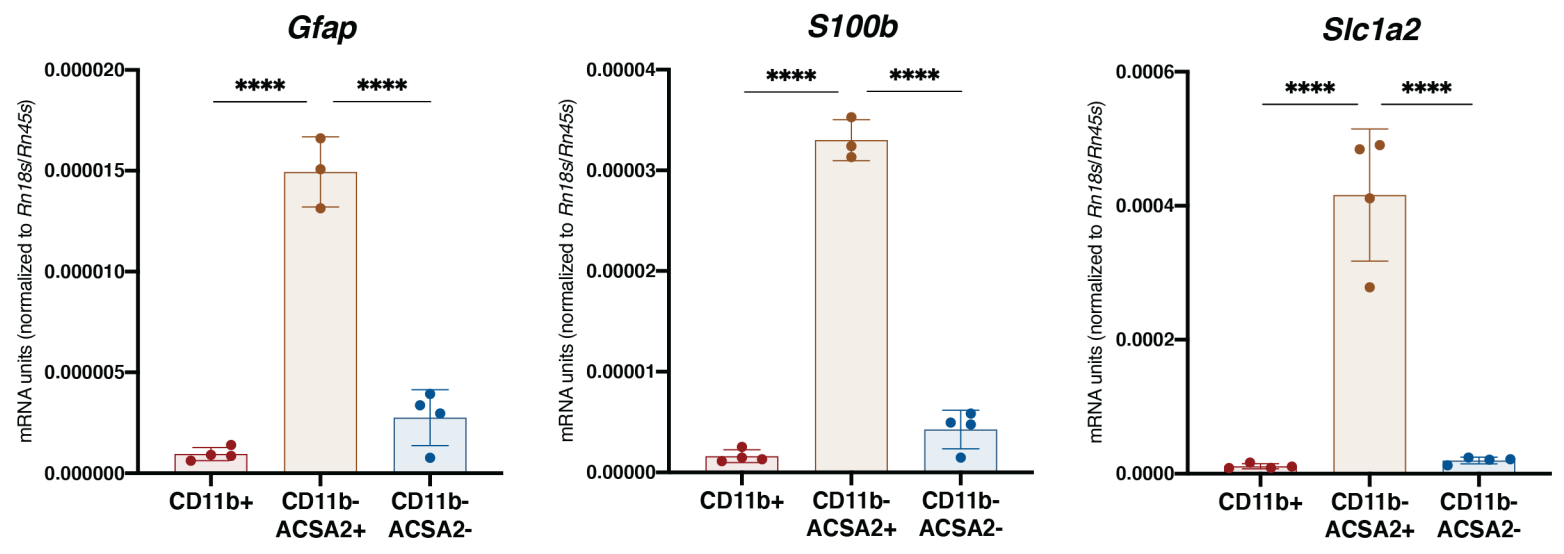

\section{Neuron markers:}
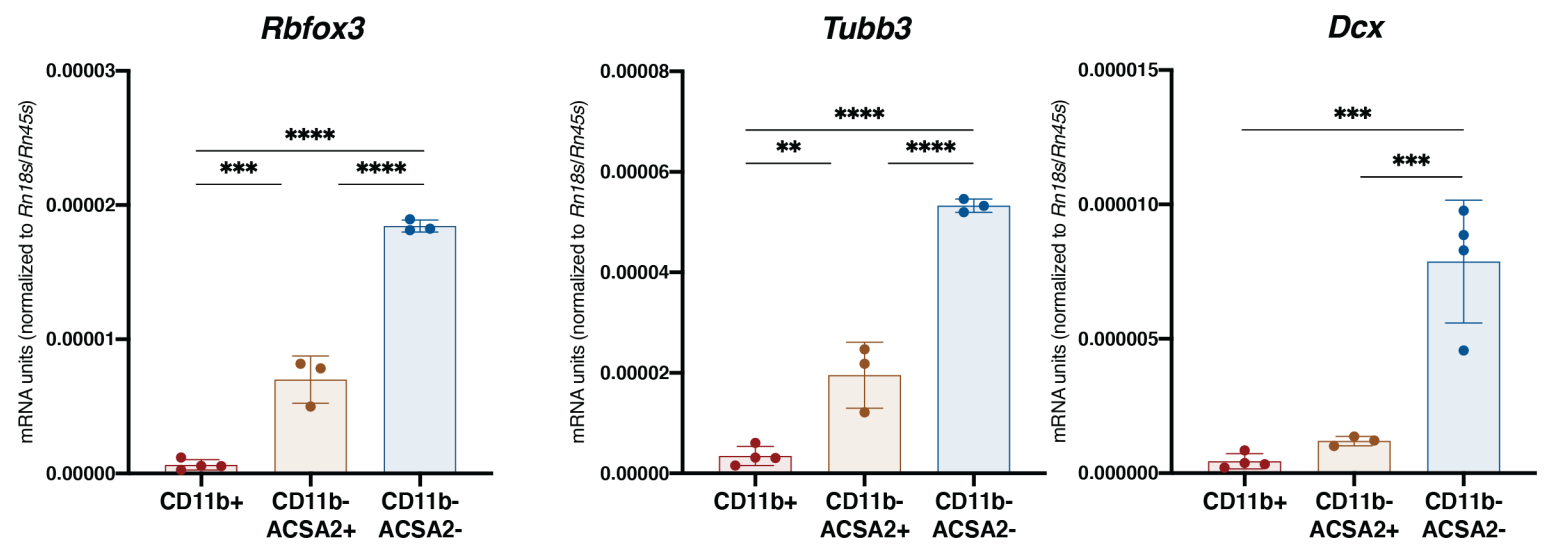

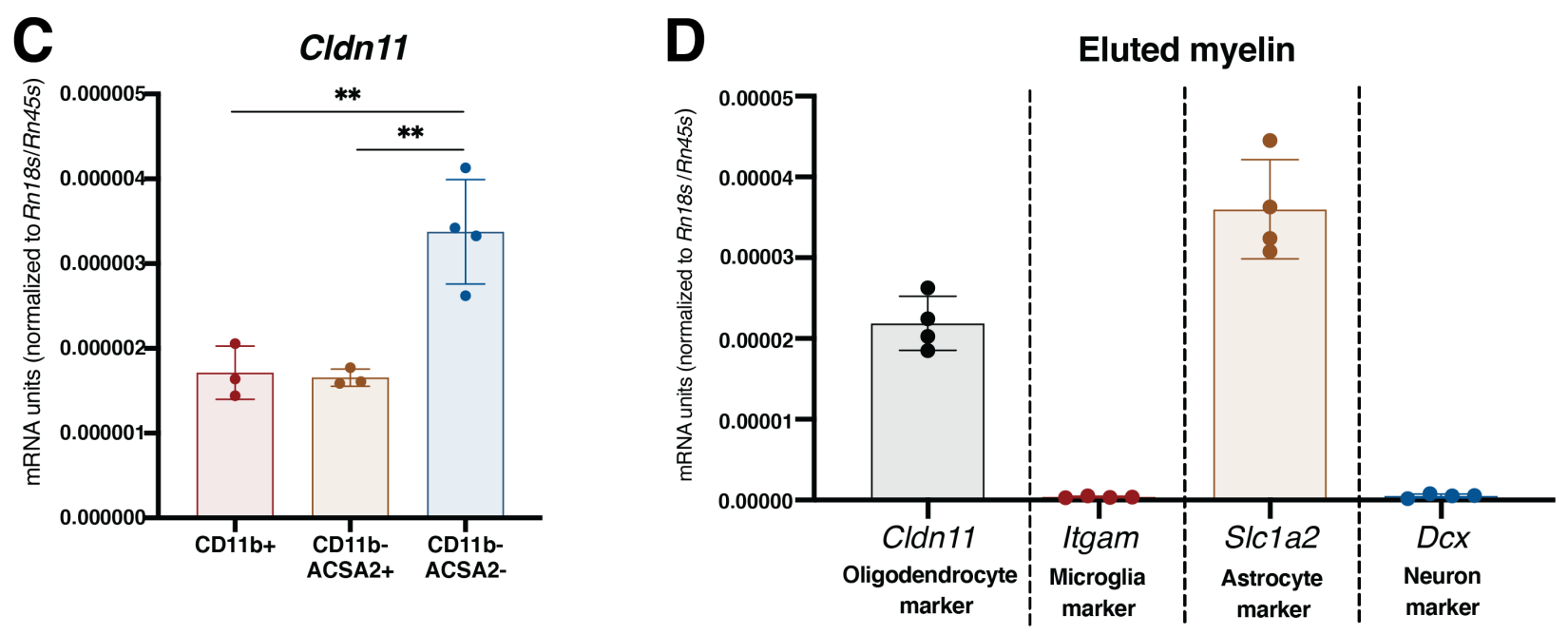

\section{Supplemental Figure 3: Expression levels of cell type-specific mRNA markers in sorted cell populations from whole adult mouse brain.}

(A, B) Gene expression analysis of indicated genes in sorted ACSA-2+ (astrocytes) and CD11b+ (microglia) populations (collected after removal of myelin via magnetic beads) as well as remaining cells, considered to be enriched for neurons. The threshold-crossing cycle $(\mathrm{Ct})$ value for each gene was normalized by the Ct value of $R n 18 s / R n 45 s$. The cell populations were each collected from 4 separate mice, with each data point representing the average of 3 technical replicates ( 3 separate qPCR wells) from each mouse. Error bars represent standard deviation among biological replicates. Statistical analysis by conducted by Ordinary one-way ANOVA followed by Bonferroni's multiple comparisons test at an $\alpha=0.05$ significance level; ${ }^{*}, p<0.5 ;{ }^{* *}, p<0.01 ;{ }^{* * *}, p<0.001 ;{ }^{* * *}, p<0.0001$. (A) Organized by population. (B) Organized by marker. (C, D) Cldn11 (protein: claudin-11, oligodendrocyte-specific protein, OSP) is used as an oligodendrocyte marker. (C) Gene expression analysis of Cldn11 in Cd11 b+ (microglia), Cd11b-/ACSA-2 ${ }^{+}$ (astrocyte) and Cd11b-/ACSA-2- (neuron) populations shows that final flow-through retains substantial Cdln11 expression compared to other populations. (D) Gene expression analysis of indicated cell type markers in eluted myelin shows that, while Cldn11 expression is higher than Itgam and Dcx, the myelin retains substantial expression of $S 100 b$ (a gene known to also be enriched in oligodendrocytes) and Slc1a2 (an astrocyte marker). 

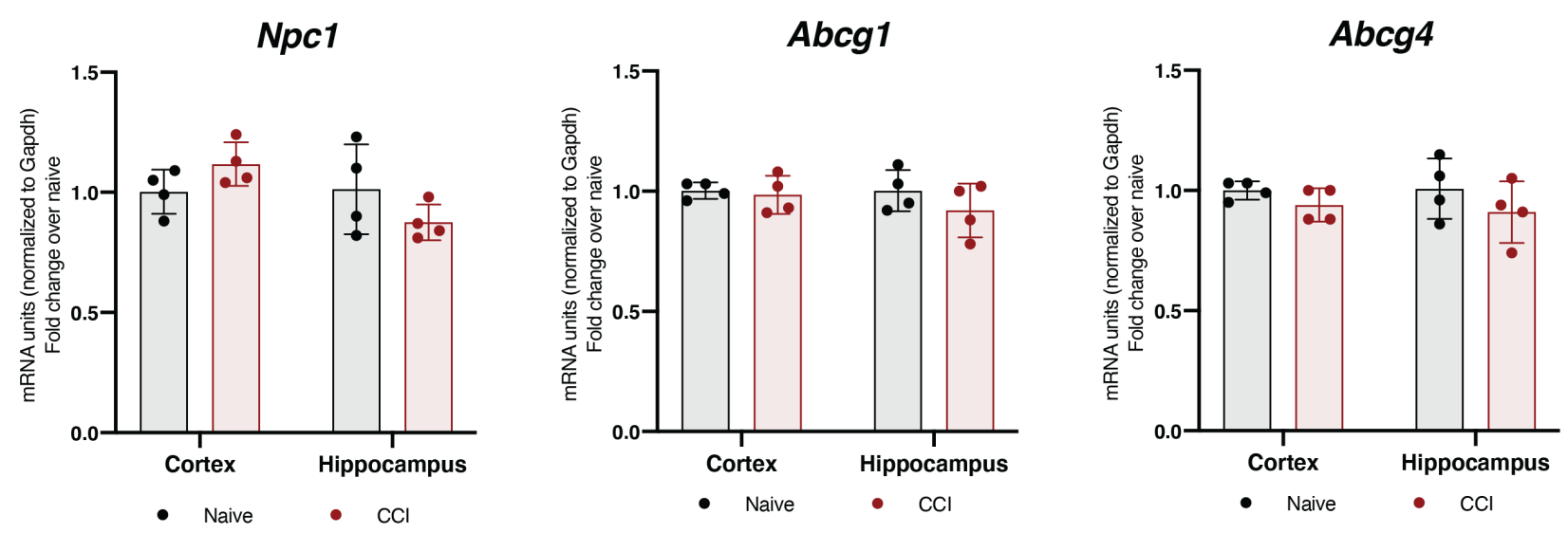

Supplemental Figure 4: Expression of additional lipid metabolic genes.

Expression of genes important in cellular lipid metabolism 3 days after injury, normalized to Gapdh. Each data point represents a separate mouse (biological replicate) and the average of 3 technical replicates. Error bars represent standard deviation. Significance assessed via T-test at $\alpha=0.05$ significance level. Gene expression of Npc1, Abcg1 and Abcg4 are unchanged following $\mathrm{CCl}$. 

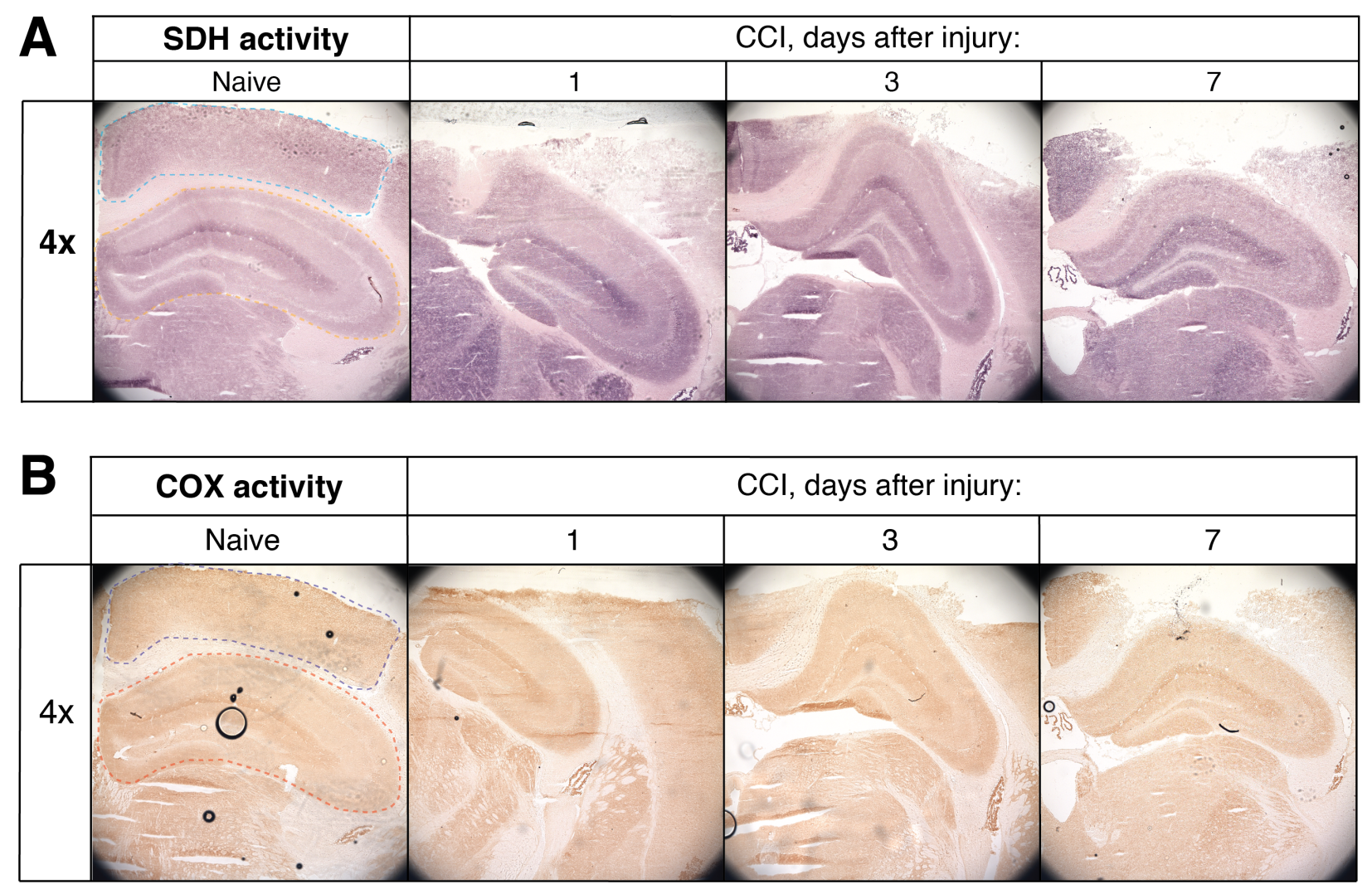
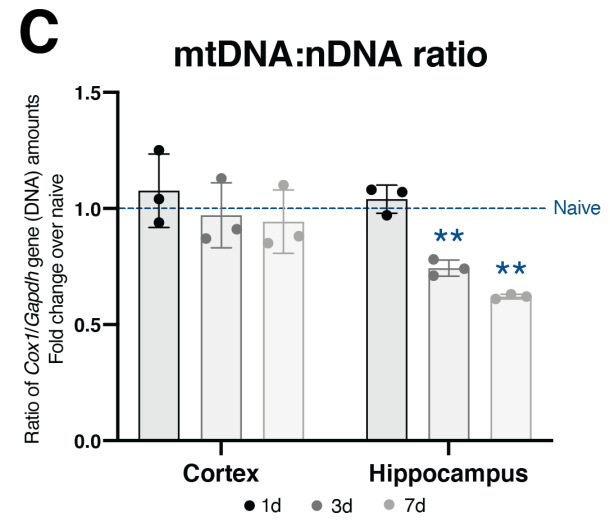

D

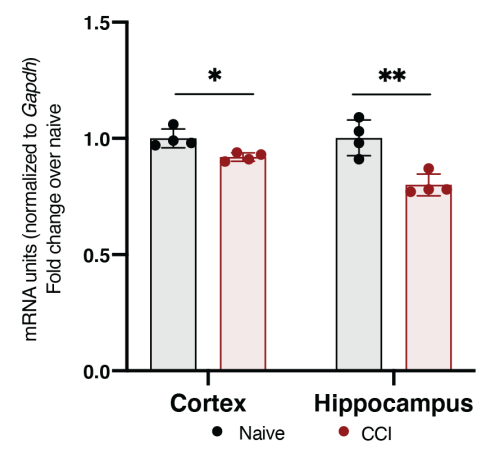

E

Cpt1a

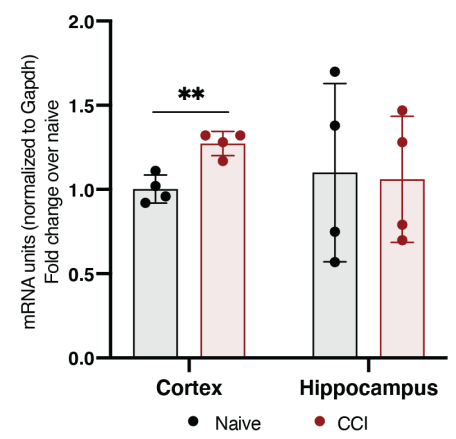



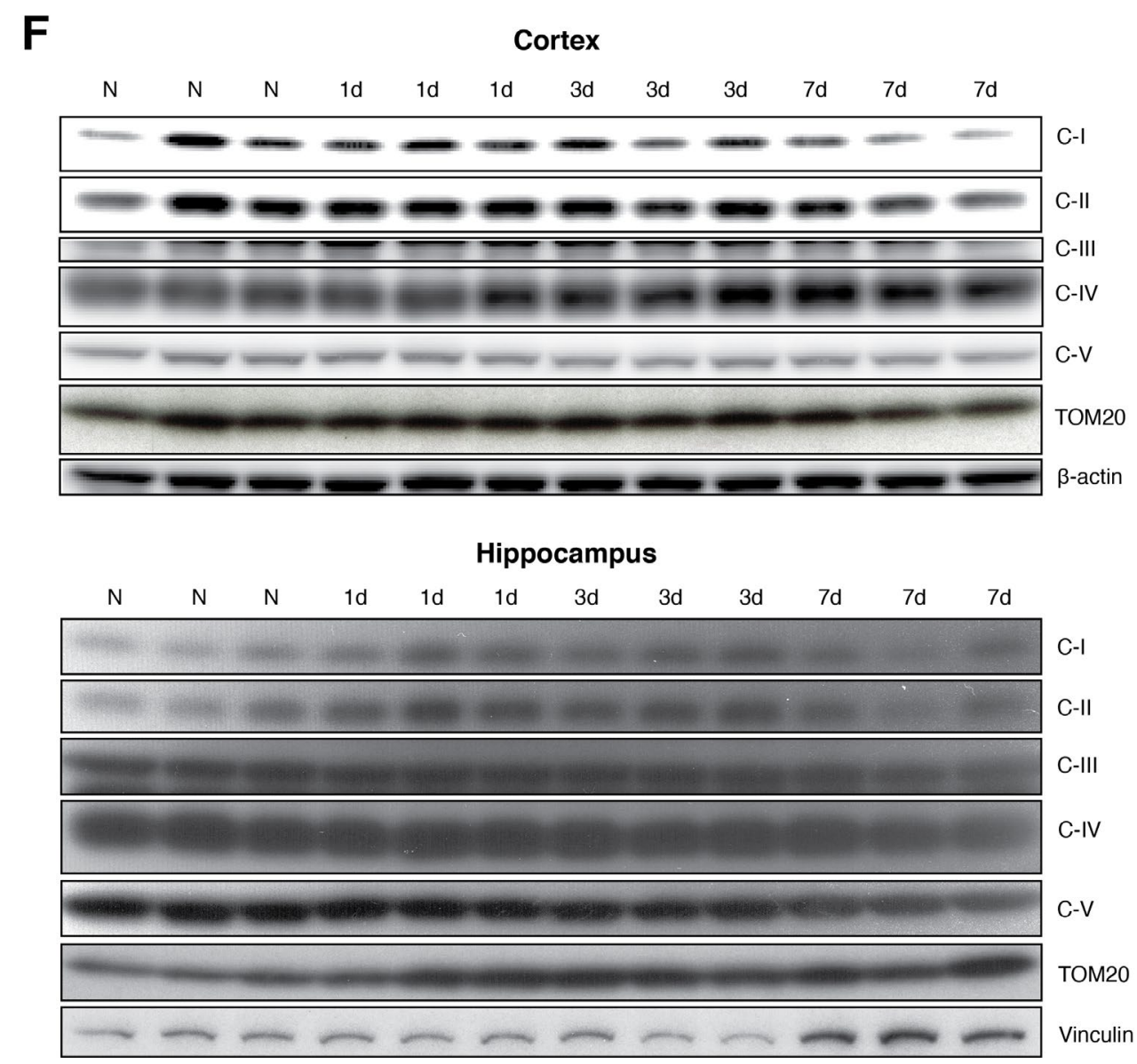

\section{Supplemental Figure 5: Additional assays related to mitochondrial respiration and mass.}

(A) Representative succinate dehydrogenase (SDH, complex-II) activity staining images in the ipsilateral cortex (outlined in blue) and ipsilateral hippocampus (outlined in yellow) at multiple time points after $\mathrm{CCl}$ injury. (B) Representative cytochrome $\mathrm{C}$ oxidase (COX) activity staining images in the ipsilateral cortex (outlined in blue) and ipsilateral hippocampus (outlined in yellow) at multiple time points after $\mathrm{CCl}$ injury. (C) Ratio of mitochondrial DNA (determined by Cox1 DNA quantity) to nuclear DNA (determined by Gapdh DNA quantity) in the ipsilateral cortex and hippocampus at indicated time-points after CCI injury. Each data point represents a separate mouse (biological replicates) and the average of 3 technical replicates. By 3 days and lasting through 7 days, the ratio was decreased in the hippocampus. (D) Gene expression analysis via qPCR of Ppargc1a, encoding PGC1 $\alpha$, a regulator of mitochondrial biogenesis, 3 days after injury. Expression was significantly reduced in both assayed brain regions. Each data point represents a separate mouse (biological replicates) and the average of 3 technical replicates (qPCR wells). Error bars represent standard deviation. *, $p<0.05$; **, $p<0.01$; T-test. (E) Gene expression of Cpt1a, which regulates mitochondrial fatty acid $\beta$-oxidation, is increased in the cortex but not in the hippocampus. (F) Western blot of OxPhos complexes in both the cortex and hippocampus in naïve and CCl tissues (1, 3 and 7 days after injury). 

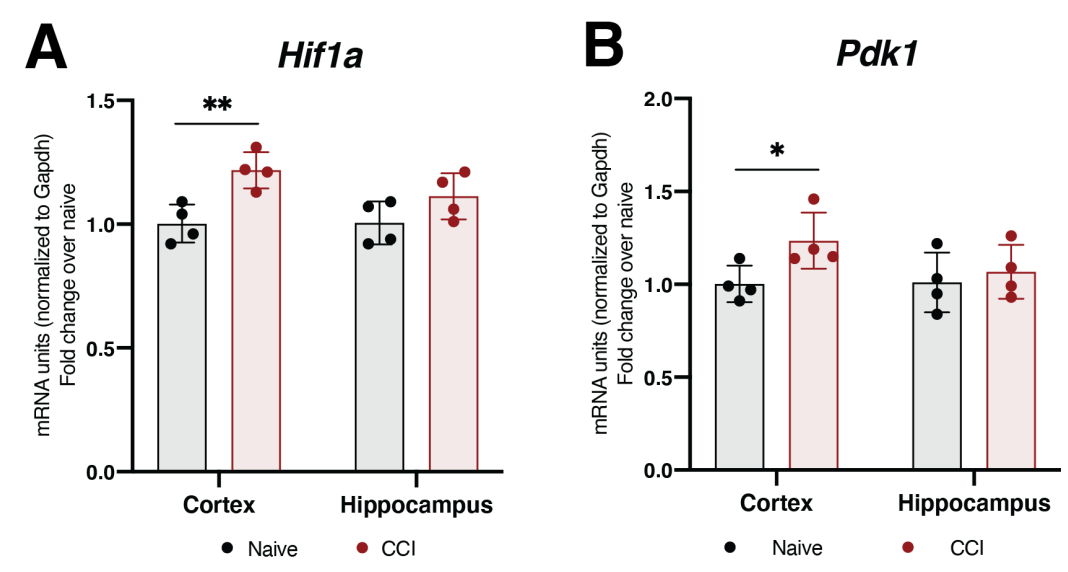

Supplemental Figure 6: Expression of genes involved in hypoxic signaling and pyruvate regulation.

These analyses were done in ipsilateral cortical and hippocampal homogenates collected 3 days after $\mathrm{CCl}$. (A) Gene expression of Hif1a, a chief mediator of the hypoxic signaling pathway, is increased in cortex with a trend for an increase in the hippocampus. (B) Gene expression of Pdk1, which encodes pyruvate dehydrogenase kinase 1 , is increased in the cortex but not in the hippocampus, suggesting that conversion of pyruvate to acetyl co-A is reduced in the cortex after $\mathrm{CCl}$. Each data point represents a separate mouse (biological replicate) and the average of 3 technical replicates (qPCR wells). Error bars represent standard deviation. *, $p<0.05 ;{ }^{* *}, p<0.01$; T-test. 


\section{Tables}

Table 1: Composition of mtDNA/nDNA ratio qPCR reaction

\begin{tabular}{|c|c|c|}
\hline Component & Volume & Sequence \\
\hline TaqMan Fast Advanced Master Mix & $10 \mu \mathrm{L}$ & \\
\hline $\begin{array}{c}\text { Gapdh TaqMan probe } \\
\text { (nuclear gene) }\end{array}$ & $1 \mu \mathrm{L}$ & $\begin{array}{c}\text { See TaqMan assay ID Mm99999915_91, } \\
\text { VIC-MGB_PL }\end{array}$ \\
\hline $\begin{array}{c}\text { Cox1 TaqMan probe } \\
\text { (mitochondrial gene) }\end{array}$ & $\begin{array}{c}0.04 \mu \mathrm{L}(200 \mathrm{nM} \\
\text { final concentration) }\end{array}$ & $\begin{array}{c}\text { FAM-TACTACTAACAGACCGCAACC- } \\
\text { MGB }\end{array}$ \\
\hline Cox1 forward primer & $\begin{array}{c}0.18 \mu \mathrm{L}(900 \mathrm{nM} \\
\text { final concentration) }\end{array}$ & 5'-TGCTAGCCGCAGGCATTACT-3' \\
\hline Cox1 reverse primer & $\begin{array}{c}0.18 \mu \mathrm{L}(900 \mathrm{nM} \\
\text { final concentration) }\end{array}$ & 5'-CGGGATCAAAGAAAGTTGTGTTT-3' \\
\hline DNA & $2 \mu \mathrm{L}(50 \mathrm{ng})$ & \\
\hline Water & $6.6 \mu \mathrm{L}$ & \\
\hline
\end{tabular}

Table 2: TaqMan probes used for bulk qPCR experiments

\begin{tabular}{|c|c|}
\hline Gene & Assay ID \\
\hline Abca1 & Mm00442646_m1 \\
\hline Abcg1 & Mm00437390_m1 \\
\hline Abcg4 & Mm00507247_m1 \\
\hline Acaca & Mm01304257_m1 \\
\hline Aif1 & Mm00479862_g1 \\
\hline Bace1 & Mm00478664_m1 \\
\hline Cd36 & Mm00432403_m1 \\
\hline Cldn11 & Mm00500915_m1 \\
\hline Cpt1a & Mm01231183_m1 \\
\hline Fasn & Mm00662319_m1 \\
\hline Gfap & Mm01253033_m1 \\
\hline Hif1a & Mm00468869_m1 \\
\hline Ldlr & Mm01177349_m1 \\
\hline Lrp1 & Mm00464608_m1 \\
\hline Npc1 & Mm00435300_m1 \\
\hline Npc2 & Mm00499230_m1 \\
\hline Pdk1 & Mm00554300_m1 \\
\hline Ppargc1a & Mm01208835_m1 \\
\hline Scarb1 & Mm00450234_m1 \\
\hline Smpd1 & Mm00488319_g1 \\
\hline Smpd2 & Mm01188195_g1 \\
\hline Smpd3 & Mm00491359_m1 \\
\hline Smpd4 & Mm00547173_m1 \\
\hline Smpd5 & Mm01205829_g1 \\
\hline Srebf1 & Mm00550338_m1 \\
\hline
\end{tabular}


Table 3: TaqMan probes used for qPCR experiments in MACS-sorted populations

\begin{tabular}{|c|c|c|}
\hline Cell type & Marker gene & Assay ID \\
\hline \multirow{3}{*}{ Astrocytes } & Gfap & Mm01253033_m1 \\
\cline { 2 - 3 } & S100b & Mm00485897_m1 \\
\cline { 2 - 3 } & Slc1a2 & Mm01275814_m1 \\
\hline \multirow{3}{*}{ Microglia } & Aif1 & Mm00479862_g1 \\
\cline { 2 - 3 } & Cx3cr1 & Mm02620111_s1 \\
\cline { 2 - 3 } & Itgam & Mm00434455_m1 \\
\hline \multirow{3}{*}{ Neurons } & Rbfox3 & Mm01248771_m1 \\
\cline { 2 - 3 } & Tubb3 & Mm00727586_s1 \\
\cline { 2 - 3 } & Dcx & Mm00438400_m1 \\
\hline Oligodendrocytes & Cldn11 & Mm00500915_m1 \\
\hline
\end{tabular}




\section{Bibliography}

[1] D. McGuire, Traumatic brain injury and neurodegenerative disease, in: M. Rizzo, S. Anderson, B. Fritzsch (Eds.), The Wiley Handbook on the Aging Mind and Brain, John Wiley \& Sons, Ltd, Chichester, UK, 2018: pp. 591-618. doi:10.1002/9781118772034.ch26.

[2] P.M. Washington, S. Villapol, M.P. Burns, Polypathology and dementia after brain trauma: Does brain injury trigger distinct neurodegenerative diseases, or should they be classified together as traumatic encephalopathy?, Exp. Neurol. 275 Pt 3 (2016) 381-388.

doi:10.1016/j.expneurol.2015.06.015.

[3] Y. Li, Y. Li, X. Li, S. Zhang, J. Zhao, X. Zhu, et al., Head Injury as a Risk Factor for Dementia and Alzheimer's Disease: A Systematic Review and Meta-Analysis of 32 Observational Studies., PLoS One. 12 (2017) e0169650. doi:10.1371/journal.pone.0169650.

[4] F. Girgis, J. Pace, J. Sweet, J.P. Miller, Hippocampal Neurophysiologic Changes after Mild Traumatic Brain Injury and Potential Neuromodulation Treatment Approaches., Front. Syst. Neurosci. 10 (2016) 8. doi:10.3389/fnsys.2016.00008.

[5] G. Halliday, Pathology and hippocampal atrophy in Alzheimer's disease., Lancet Neurol. 16 (2017) 862-864. doi:10.1016/S1474-4422(17)30343-5.

[6] P.P. Tsitsopoulos, N. Marklund, Amyloid- $\beta$ Peptides and Tau Protein as Biomarkers in Cerebrospinal and Interstitial Fluid Following Traumatic Brain Injury: A Review of Experimental and Clinical Studies., Front. Neurol. 4 (2013) 79. doi:10.3389/fneur.2013.00079.

[7] P.M. Washington, P.A. Forcelli, T. Wilkins, D.N. Zapple, M. Parsadanian, M.P. Burns, The effect of injury severity on behavior: a phenotypic study of cognitive and emotional deficits after mild, moderate, and severe controlled cortical impact injury in mice., J. Neurotrauma. 29 (2012) 22832296. doi:10.1089/neu.2012.2456.

[8] M.D. Ikonomovic, K. Uryu, E.E. Abrahamson, J.R. Ciallella, J.Q. Trojanowski, V.M.-Y. Lee, et al., Alzheimer's pathology in human temporal cortex surgically excised after severe brain injury., Exp. Neurol. 190 (2004) 192-203. doi:10.1016/j.expneurol.2004.06.011.

[9] M. Prins, T. Greco, D. Alexander, C.C. Giza, The pathophysiology of traumatic brain injury at a glance., Dis. Model. Mech. 6 (2013) 1307-1315. doi:10.1242/dmm.011585.

[10] S.Y. Ng, A.Y.W. Lee, Traumatic brain injuries: pathophysiology and potential therapeutic targets., Front. Cell Neurosci. 13 (2019) 528. doi:10.3389/fncel.2019.00528.

[11] R.R. Agrawal, J. Montesinos, D. Larrea, E. Area-Gomez, M. Pera, The silence of the fats: A MAM's story about Alzheimer., Neurobiol. Dis. 145 (2020) 105062. doi:10.1016/j.nbd.2020.105062.

[12] J.H. Kim, S.M. Ee, J. Jittiwat, E.S. Ong, A.A. Farooqui, A.M. Jenner, et al., Increased expression of acyl-coenzyme A: cholesterol acyltransferase- 1 and elevated cholesteryl esters in the hippocampus after excitotoxic injury., Neuroscience. 185 (2011) 125-134. doi:10.1016/j.neuroscience.2011.04.018.

[13] A. Roux, L. Muller, S.N. Jackson, J. Post, K. Baldwin, B. Hoffer, et al., Mass spectrometry imaging 
of rat brain lipid profile changes over time following traumatic brain injury., J. Neurosci. Methods. 272 (2016) 19-32. doi:10.1016/j.jneumeth.2016.02.004.

[14] M. Pera, D. Larrea, C. Guardia-Laguarta, J. Montesinos, K.R. Velasco, R.R. Agrawal, et al., Increased localization of APP-C99 in mitochondria-associated ER membranes causes mitochondrial dysfunction in Alzheimer disease., EMBO J. 36 (2017) 3356-3371. doi:10.15252/embj.201796797.

[15] J. Montesinos, M. Pera, D. Larrea, C. Guardia-Laguarta, R.R. Agrawal, K.R. Velasco, et al., The Alzheimer's disease-associated C99 fragment of APP regulates cellular cholesterol trafficking., EMBO J. 39 (2020) e103791. doi:10.15252/embj.2019103791.

[16] J.E. Vance, MAM (mitochondria-associated membranes) in mammalian cells: lipids and beyond., Biochim. Biophys. Acta. 1841 (2014) 595-609. doi:10.1016/j.bbalip.2013.11.014.

[17] E. Area-Gomez, M. Del Carmen Lara Castillo, M.D. Tambini, C. Guardia-Laguarta, A.J.C. de Groof, M. Madra, et al., Upregulated function of mitochondria-associated ER membranes in Alzheimer disease., EMBO J. 31 (2012) 4106-4123. doi:10.1038/emboj.2012.202.

[18] R.M. Adibhatla, J.F. Hatcher, Role of lipids in brain injury and diseases., Future Lipidol. 2 (2007) 403-422. doi:10.2217/17460875.2.4.403.

[19] X. Ma, A. Aravind, B.J. Pfister, N. Chandra, J. Haorah, Animal models of traumatic brain injury and assessment of injury severity., Mol. Neurobiol. 56 (2019) 5332-5345. doi:10.1007/s12035-0181454-5.

[20] N.D. Osier, C.E. Dixon, The controlled cortical impact model: applications, considerations for researchers, and future directions., Front. Neurol. 7 (2016) 134. doi:10.3389/fneur.2016.00134.

[21] R.M. Bryan, L. Cherian, C. Robertson, Regional cerebral blood flow after controlled cortical impact injury in rats., Anesth. Analg. 80 (1995) 687-695. doi:10.1097/00000539-199504000-00007.

[22] X.-H. Chen, R. Siman, A. Iwata, D.F. Meaney, J.Q. Trojanowski, D.H. Smith, Long-term accumulation of amyloid-beta, beta-secretase, presenilin-1, and caspase-3 in damaged axons following brain trauma., Am. J. Pathol. 165 (2004) 357-371. doi:10.1016/s0002-9440(10)63303-2.

[23] L.C. Schmued, C.C. Stowers, A.C. Scallet, L. Xu, Fluoro-Jade $C$ results in ultra high resolution and contrast labeling of degenerating neurons., Brain Res. 1035 (2005) 24-31. doi:10.1016/j.brainres.2004.11.054.

[24] L.F. Eng, R.S. Ghirnikar, GFAP and astrogliosis., Brain Pathol. 4 (1994) 229-237.

[25] E. Postler, A. Rimner, R. Beschorner, H.J. Schluesener, R. Meyermann, "Allograft-inflammatoryfactor-1 is upregulated in microglial cells in human cerebral infarctions"., J. Neuroimmunol. 108 (2000) 244-250. doi:10.1016/s0165-5728(00)00283-6.

[26] J.M. Bronstein, S. Tiwari-Woodruff, A.G. Buznikov, D.B. Stevens, Involvement of OSP/claudin-11 in oligodendrocyte membrane interactions: role in biology and disease., J. Neurosci. Res. 59 (2000) 706-711. doi:10.1002/(SICl)1097-4547(20000315)59:6<706::AID-JNR2>3.0.CO;2-D.

[27] J.E. Vance, Phospholipid synthesis in a membrane fraction associated with mitochondria., J. Biol. Chem. 265 (1990) 7248-7256. 
[28] J.E. Vance, Phosphatidylserine and phosphatidylethanolamine in mammalian cells: two metabolically related aminophospholipids., J. Lipid Res. 49 (2008) 1377-1387. doi:10.1194/jlr.R700020-JLR200.

[29] J.P. Slotte, Sphingomyelin-cholesterol interactions in biological and model membranes., Chem Phys Lipids. 102 (1999) 13-27. doi:10.1016/s0009-3084(99)00071-7.

[30] B.X. Wu, V. Rajagopalan, P.L. Roddy, C.J. Clarke, Y.A. Hannun, Identification and characterization of murine mitochondria-associated neutral sphingomyelinase (MA-nSMase), the mammalian sphingomyelin phosphodiesterase 5., J. Biol. Chem. 285 (2010) 17993-18002. doi:10.1074/jbc.M110.102988.

[31] A. Mehlem, C.E. Hagberg, L. Muhl, U. Eriksson, A. Falkevall, Imaging of neutral lipids by oil red O for analyzing the metabolic status in health and disease., Nat. Protoc. 8 (2013) 1149-1154. doi:10.1038/nprot.2013.055.

[32] L. Shi, C. Zheng, Y. Shen, Z. Chen, E.S. Silveira, L. Zhang, et al., Optical imaging of metabolic dynamics in animals., Nat. Commun. 9 (2018) 2995. doi:10.1038/s41467-018-05401-3.

[33] W.S. Kim, C.S. Weickert, B. Garner, Role of ATP-binding cassette transporters in brain lipid transport and neurological disease., J. Neurochem. 104 (2008) 1145-1166. doi:10.1111/j.14714159.2007.05099.x.

[34] I.J. Goldberg, R.H. Eckel, N.A. Abumrad, Regulation of fatty acid uptake into tissues: lipoprotein lipase- and CD36-mediated pathways., J. Lipid Res. 50 Suppl (2009) S86-90. doi:10.1194/jlr.R800085-JLR200.

[35] S. Grampp, V. Schmid, R. Salama, V. Lauer, F. Kranz, J.L. Platt, et al., Multiple renal cancer susceptibility polymorphisms modulate the HIF pathway., PLoS Genet. 13 (2017) e1006872. doi:10.1371/journal.pgen.1006872.

[36] E.M. Foley, J.D. Esko, Hepatic Heparan Sulfate Proteoglycans and Endocytic Clearance of Triglyceride-Rich Lipoproteins, in: Glycosaminoglycans in Development, Health and Disease, Elsevier, 2010: pp. 213-233. doi:10.1016/S1877-1173(10)93010-X.

[37] R.E. Infante, M.L. Wang, A. Radhakrishnan, H.J. Kwon, M.S. Brown, J.L. Goldstein, NPC2 facilitates bidirectional transfer of cholesterol between NPC1 and lipid bilayers, a step in cholesterol egress from lysosomes., Proc. Natl. Acad. Sci. USA. 105 (2008) 15287-15292. doi:10.1073/pnas.0807328105.

[38] A. Habib, D. Chokr, J. Wan, P. Hegde, M. Mabire, M. Siebert, et al., Inhibition of monoacylglycerol lipase, an anti-inflammatory and antifibrogenic strategy in the liver., Gut. 68 (2019) 522-532. doi:10.1136/gutjnl-2018-316137.

[39] J.D. Horton, J.L. Goldstein, M.S. Brown, SREBPs: activators of the complete program of cholesterol and fatty acid synthesis in the liver., J. Clin. Invest. 109 (2002) 1125-1131. doi:10.1172/JCl15593.

[40] F. Ameer, L. Scandiuzzi, S. Hasnain, H. Kalbacher, N. Zaidi, De novo lipogenesis in health and disease., Metab. Clin. Exp. 63 (2014) 895-902. doi:10.1016/j.metabol.2014.04.003. 
[41] L. Helming, J. Winter, S. Gordon, The scavenger receptor CD36 plays a role in cytokine-induced macrophage fusion., J. Cell Sci. 122 (2009) 453-459. doi:10.1242/jcs.037200.

[42] G. Cheng, R. Kong, L. Zhang, J. Zhang, Mitochondria in traumatic brain injury and mitochondrialtargeted multipotential therapeutic strategies., Br. J. Pharmacol. 167 (2012) 699-719. doi:10.1111/j.1476-5381.2012.02025.x.

[43] J.B. Hiebert, Q. Shen, A.R. Thimmesch, J.D. Pierce, Traumatic brain injury and mitochondrial dysfunction., Am. J. Med. Sci. 350 (2015) 132-138. doi:10.1097/MAJ.0000000000000506.

[44] R.R. Agrawal, K.A. Tamucci, M. Pera, D. Larrea, Assessing mitochondrial respiratory bioenergetics in whole cells and isolated organelles by microplate respirometry., Methods Cell Biol. 155 (2020) 157-180. doi:10.1016/bs.mcb.2019.12.005.

[45] J.M. Ross, Visualization of mitochondrial respiratory function using cytochrome c oxidase/succinate dehydrogenase (COX/SDH) double-labeling histochemistry., J. Vis. Exp. (2011) e3266. doi:10.3791/3266.

[46] F.R. Jornayvaz, G.I. Shulman, Regulation of mitochondrial biogenesis., Essays Biochem. 47 (2010) 69-84. doi:10.1042/bse0470069.

[47] E. Maloney-Wilensky, V. Gracias, A. Itkin, K. Hoffman, S. Bloom, W. Yang, et al., Brain tissue oxygen and outcome after severe traumatic brain injury: a systematic review., Crit. Care Med. 37 (2009) 2057-2063. doi:10.1097/CCM.0b013e3181a009f8.

[48] D.C. Fuhrmann, C. Olesch, N. Kurrle, F. Schnütgen, S. Zukunft, I. Fleming, et al., Chronic Hypoxia Enhances $\beta$-Oxidation-Dependent Electron Transport via Electron Transferring Flavoproteins., Cells. 8 (2019). doi:10.3390/cells8020172.

[49] M.K. Jha, S. Jeon, K. Suk, Pyruvate Dehydrogenase Kinases in the Nervous System: Their Principal Functions in Neuronal-glial Metabolic Interaction and Neuro-metabolic Disorders., Curr Neuropharmacol. 10 (2012) 393-403. doi:10.2174/157015912804143586.

[50] N. Longo, M. Frigeni, M. Pasquali, Carnitine transport and fatty acid oxidation., Biochim. Biophys. Acta. 1863 (2016) 2422-2435. doi:10.1016/j.bbamcr.2016.01.023.

[51] J.D. McGarry, N.F. Brown, The mitochondrial carnitine palmitoyltransferase system. From concept to molecular analysis., Eur. J. Biochem. 244 (1997) 1-14. doi:10.1111/j.1432-1033.1997.00001.x.

[52] J.E. Vance, R.B. Campenot, D.E. Vance, The synthesis and transport of lipids for axonal growth and nerve regeneration., Biochim. Biophys. Acta. 1486 (2000) 84-96. doi:10.1016/s13881981(00)00050-0.

[53] D.H. Smith, X.-H. Chen, A. Iwata, D.I. Graham, Amyloid beta accumulation in axons after traumatic brain injury in humans., J. Neurosurg. 98 (2003) 1072-1077. doi:10.3171/jns.2003.98.5.1072.

[54] C.M. Cartagena, A. Mountney, H. Hwang, A. Swiercz, Z. Rammelkamp, A.M. Boutte, et al., Subacute Changes in Cleavage Processing of Amyloid Precursor Protein and Tau following Penetrating Traumatic Brain Injury., PLoS One. 11 (2016) e0158576. doi:10.1371/journal.pone.0158576.

[55] T. Seo, P.M. Oelkers, M.R. Giattina, T.S. Worgall, S.L. Sturley, R.J. Deckelbaum, Differential 
modulation of ACAT1 and ACAT2 transcription and activity by long chain free fatty acids in cultured cells., Biochemistry. 40 (2001) 4756-4762. doi:10.1021/bi0022947.

[56] J.G. Wood, R.M. Dawson, Lipid and protein changes in sciatic nerve during Wallerian degeneration., J. Neurochem. 22 (1974) 631-635. doi:10.1111/j.1471-4159.1974.tb04274.x.

[57] J.K. Yao, V. Natarajan, P.J. Dyck, The sequential alterations of endoneurial cholesterol and fatty acid in Wallerian degeneration and regeneration., J. Neurochem. 35 (1980) 933-940. doi:10.1111/j.1471-4159.1980.tb07092.x.

[58] E.I. de Chaves, A.E. Rusiñol, D.E. Vance, R.B. Campenot, J.E. Vance, Role of lipoproteins in the delivery of lipids to axons during axonal regeneration., J. Biol. Chem. 272 (1997) 30766-30773. doi:10.1074/jbc.272.49.30766.

[59] C.M. Cartagena, F. Ahmed, M.P. Burns, A. Pajoohesh-Ganji, D.T. Pak, A.I. Faden, et al., Cortical injury increases cholesterol 24S hydroxylase (Cyp46) levels in the rat brain., J. Neurotrauma. 25 (2008) 1087-1098. doi:10.1089/neu.2007.0444.

[60] D. Tweedie, L. Rachmany, D.S. Kim, V. Rubovitch, E. Lehrmann, Y. Zhang, et al., Mild traumatic brain injury-induced hippocampal gene expressions: The identification of target cellular processes for drug development., J. Neurosci. Methods. 272 (2016) 4-18. doi:10.1016/j.jneumeth.2016.02.003.

[61] D.J. Loane, P.M. Washington, L. Vardanian, A. Pocivavsek, H.-S. Hoe, K.E. Duff, et al., Modulation of $A B C A 1$ by an LXR agonist reduces $\beta$-amyloid levels and improves outcome after traumatic brain injury., J. Neurotrauma. 28 (2011) 225-236. doi:10.1089/neu.2010.1595.

[62] S.B. Jasmin, V. Pearson, D. Lalonde, D. Domenger, L. Théroux, J. Poirier, Differential regulation of $A B C A 1$ and $A B C G 1$ gene expressions in the remodeling mouse hippocampus after entorhinal cortex lesion and liver-X receptor agonist treatment., Brain Res. 1562 (2014) 39-51. doi:10.1016/j.brainres.2014.03.016.

[63] J. Marschallinger, T. Iram, M. Zardeneta, S.E. Lee, B. Lehallier, M.S. Haney, et al., Lipid-dropletaccumulating microglia represent a dysfunctional and proinflammatory state in the aging brain., Nat. Neurosci. 23 (2020) 194-208. doi:10.1038/s41593-019-0566-1.

[64] J.K. Boyles, C.D. Zoellner, L.J. Anderson, L.M. Kosik, R.E. Pitas, K.H. Weisgraber, et al., A role for apolipoprotein E, apolipoprotein A-I, and low density lipoprotein receptors in cholesterol transport during regeneration and remyelination of the rat sciatic nerve., J. Clin. Invest. 83 (1989) 10151031. doi:10.1172/JCl113943.

[65] J.F. Goodrum, T. Earnhardt, N. Goines, T.W. Bouldin, Fate of myelin lipids during degeneration and regeneration of peripheral nerve: an autoradiographic study., J. Neurosci. 14 (1994) 357-367.

[66] D.C. Barbacci, A. Roux, L. Muller, S.N. Jackson, J. Post, K. Baldwin, et al., Mass spectrometric imaging of ceramide biomarkers tracks therapeutic response in traumatic brain injury., ACS Chem. Neurosci. 8 (2017) 2266-2274. doi:10.1021/acschemneuro.7b00189.

[67] S. Chatterjee, Neutral sphingomyelinase action stimulates signal transduction of tumor necrosis factor-alpha in the synthesis of cholesteryl esters in human fibroblasts., J. Biol. Chem. 269 (1994) 
879-882.

[68] S. Jayadev, C.M. Linardic, Y.A. Hannun, Identification of arachidonic acid as a mediator of sphingomyelin hydrolysis in response to tumor necrosis factor alpha., J. Biol. Chem. 269 (1994) 5757-5763.

[69] S.A. Novgorodov, J.R. Voltin, M.A. Gooz, L. Li, J.J. Lemasters, T.I. Gudz, Acid sphingomyelinase promotes mitochondrial dysfunction due to glutamate-induced regulated necrosis., J. Lipid Res. 59 (2018) 312-329. doi:10.1194/jlr.M080374.

[70] Y. Zhang, K. Chen, S.A. Sloan, M.L. Bennett, A.R. Scholze, S. O'Keeffe, et al., An RNAsequencing transcriptome and splicing database of glia, neurons, and vascular cells of the cerebral cortex., J. Neurosci. 34 (2014) 11929-11947. doi:10.1523/JNEUROSCI.1860-14.2014.

[71] M.S. Köberlin, B. Snijder, L.X. Heinz, C.L. Baumann, A. Fauster, G.I. Vladimer, et al., A conserved circular network of coregulated lipids modulates innate immune responses., Cell. 162 (2015) 170183. doi:10.1016/j.cell.2015.05.051.

[72] J.W. Kinney, S.M. Bemiller, A.S. Murtishaw, A.M. Leisgang, A.M. Salazar, B.T. Lamb, Inflammation as a central mechanism in Alzheimer's disease., Alzheimers Dement (N Y). 4 (2018) 575-590. doi:10.1016/j.trci.2018.06.014.

[73] K.R. Feingold, J.K. Shigenaga, M.R. Kazemi, C.M. McDonald, S.M. Patzek, A.S. Cross, et al., Mechanisms of triglyceride accumulation in activated macrophages., J. Leukoc. Biol. 92 (2012) 829-839. doi:10.1189/jlb.1111537.

[74] C.N. Barber, D.M. Raben, Lipid metabolism crosstalk in the brain: glia and neurons., Front. Cell Neurosci. 13 (2019) 212. doi:10.3389/fncel.2019.00212.

[75] X. Yang, W. Sheng, G.Y. Sun, J.C.-M. Lee, Effects of fatty acid unsaturation numbers on membrane fluidity and $\alpha$-secretase-dependent amyloid precursor protein processing., Neurochem. Int. 58 (2011) 321-329. doi:10.1016/j.neuint.2010.12.004.

[76] J. Hardy, Membrane damage is at the core of Alzheimer's disease., Lancet Neurol. 16 (2017) 342. doi:10.1016/S1474-4422(17)30091-1.

[77] H. Kuwata, S. Hara, Role of acyl-CoA synthetase ACSL4 in arachidonic acid metabolism., Prostaglandins Other Lipid Mediat. 144 (2019) 106363. doi:10.1016/j.prostaglandins.2019.106363.

[78] R. Chouinard-Watkins, R.P. Bazinet, ACSL6 is critical for maintaining brain DHA levels., Proc. Natl. Acad. Sci. USA. 115 (2018) 12343-12345. doi:10.1073/pnas.1817557115.

[79] T.M. Lewin, C.G. Van Horn, S.K. Krisans, R.A. Coleman, Rat liver acyl-CoA synthetase 4 is a peripheral-membrane protein located in two distinct subcellular organelles, peroxisomes, and mitochondrial-associated membrane, Arch. Biochem. Biophys. 404 (2002) 263-270. doi:10.1016/S0003-9861(02)00247-3.

[80] J. Montesinos, C. Guardia-Laguarta, E. Area-Gomez, The fat brain., Curr. Opin. Clin. Nutr. Metab. Care. 23 (2020) 68-75. doi:10.1097/MCO.0000000000000634.

[81] T.J. Kilbaugh, M. Karlsson, M. Byro, A. Bebee, J. Ralston, S. Sullivan, et al., Mitochondrial bioenergetic alterations after focal traumatic brain injury in the immature brain., Exp. Neurol. 271 
(2015) 136-144. doi:10.1016/j.expneurol.2015.05.009.

[82] T.J. Kilbaugh, M. Karlsson, A.-C. Duhaime, M.J. Hansson, E. Elmer, S.S. Margulies, Mitochondrial response in a toddler-aged swine model following diffuse non-impact traumatic brain injury., Mitochondrion. 26 (2016) 19-25. doi:10.1016/j.mito.2015.11.001.

[83] A.S. Divakaruni, M. Wallace, C. Buren, K. Martyniuk, A.Y. Andreyev, E. Li, et al., Inhibition of the mitochondrial pyruvate carrier protects from excitotoxic neuronal death., J. Cell Biol. 216 (2017) 1091-1105. doi:10.1083/jcb.201612067.

[84] R.B. Chan, T.G. Oliveira, E.P. Cortes, L.S. Honig, K.E. Duff, S.A. Small, et al., Comparative lipidomic analysis of mouse and human brain with Alzheimer disease., J. Biol. Chem. 287 (2012) 2678-2688. doi:10.1074/jbc.M111.274142.

[85] E. Area-Gomez, Assessing the function of mitochondria-associated ER membranes., Meth. Enzymol. 547 (2014) 181-197. doi:10.1016/B978-0-12-801415-8.00011-4.

[86] J. Montesinos, E. Area-Gomez, M. Schlame, Analysis of phospholipid synthesis in mitochondria., Methods Cell Biol. 155 (2020) 321-335. doi:10.1016/bs.mcb.2019.12.003.

[87] J. Montesinos, E. Area-Gomez, Isolation of mitochondria-associated ER membranes., Methods Cell Biol. 155 (2020) 33-44. doi:10.1016/bs.mcb.2019.12.001. 